\title{
Shaping the Epigenetic Landscape: Complexities and Consequences
}

\author{
Megan A. Coomera, ${ }^{a}$, Lucy Ham ${ }^{\mathrm{b}, 1}$, Michael P. H. Stumpf $f^{\mathrm{a}, \mathrm{b}, *}$ \\ ${ }^{a}$ School of Mathematics and Statistics, University of Melbourne, Parkville VIC 3010, Australia \\ ${ }^{b}$ School of BioSciences, University of Melbourne, Parkville VIC 3010, Australia
}

\begin{abstract}
The metaphor of the Waddington epigenetic landscape has become an iconic representation of the cellular differentiation process. Recent accessibility of single-cell transcriptomic data has provided new opportunities for quantifying this originally conceptual tool that could offer insight into the gene regulatory networks underlying cellular development. While a number of methods for constructing the landscape have been proposed, by far the most commonly employed approach is based on computing the landscape as the negative logarithm of the steady-state probability distribution. Here, we use a simple model to highlight the complexities and limitations that arise when reconstructing the potential landscape in the presence of stochastic fluctuations. We consider how the landscape changes in accordance with different stochastic systems, and show that it is the subtle interplay between the deterministic and stochastic components of the system that ultimately shapes the landscape. We further discuss how the presence of noise has important implications for the identifiability of the regulatory dynamics from experimental data.
\end{abstract}

Keywords: epigenentic landscape, potential, stochastic dynamical systems, bifurcations

\section{Introduction}

Waddington's epigenetic landscape has become more than just a metaphor for cellular differentiation and developmental biology. In recent years it has garnered renewed interest due to (i) technological advances in e.g. single cell biology; and (ii) because of its close, intimate link to mathematical models that describe developmental processes in biology. Due to the excellent expository work of [1] and [2], but also through Waddington's own writing and influence [3, the Waddington (also known as epigenetic) landscape, has made mathematical concepts palatable to traditionally non-mathematical audiences. The intuitive representation of a dynamical system provided by the landscape also appeals to mathematical scientists, and has done so for some time. Réne Thom was very interested in developmental biology 4 and communicated extensively with Waddington.

One of the appeals of the landscape is that it offers a useful way of making mathematical concepts accessible. Traditionally, however, the analysis (and interpretation) of Waddington's epigenetic landscape has revolved around considering deterministic dynamics. At the cellular level, however, noise - caused by the stochasticity underlying the molecular processes involved in gene regulation, protein turn-over etc - is ubiquitous. Noise interacts with dynamics in often non-intuitive ways [5], and it is important to set out the conditions under which noisy and determin-

\footnotetext{
*Correspondence: mstumpf@unimelb.edu.au

${ }^{1}$ These authors contributed equally to this work
}

istic dynamics agree with respect to important qualitative (and quantitative) aspects of the dynamics.

Linking the landscape to gradient systems, deterministic dynamical systems of the type

$$
\frac{d x}{d t}=f(x)=-\nabla U(x),
$$

where $x$ denotes the state of the system, and where $U(x)$ is a potential function, has proven to be particularly fruitful. For such systems we can access powerful mathematical frameworks, such as Morse theory (every gradient system is a Morse function) [6, 7]; and a slew of further mathematical developments following Morse's original work (for a general overview see $[8,9]$ ). On the face of it, restricting our attention to gradient systems may appear limiting. But there are two important results, that mean that this is in fact not the case: (i) gradient systems (or more generally, Morse-Smale systems) are very common, and any function chosen at random is a Morse function; (ii) systems that are not gradient systems can be transformed into gradient systems in well understood ways. Thus gradient systems are (qualitatively and certain aspects at least semiquantitatively) representative of more general dynamical systems 6, 7.

Noisy biological systems are frequently modelled in terms of stochastic differential equations (SDEs) [10. Here the state of the system $x$, usually defined in terms of molecular concentrations, is described by

$$
d x=f(x) d t+g(x) d W_{t},
$$

where $f(x)$ represents the deterministic dynamics of the system and $g(x) d W_{t}$ the stochastic dynamics, with $d W_{t}$ 
A.

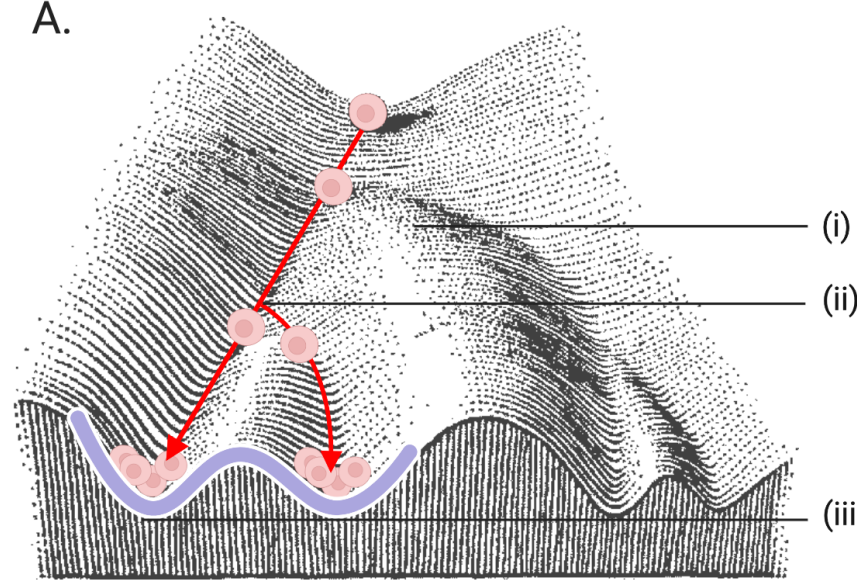

B.

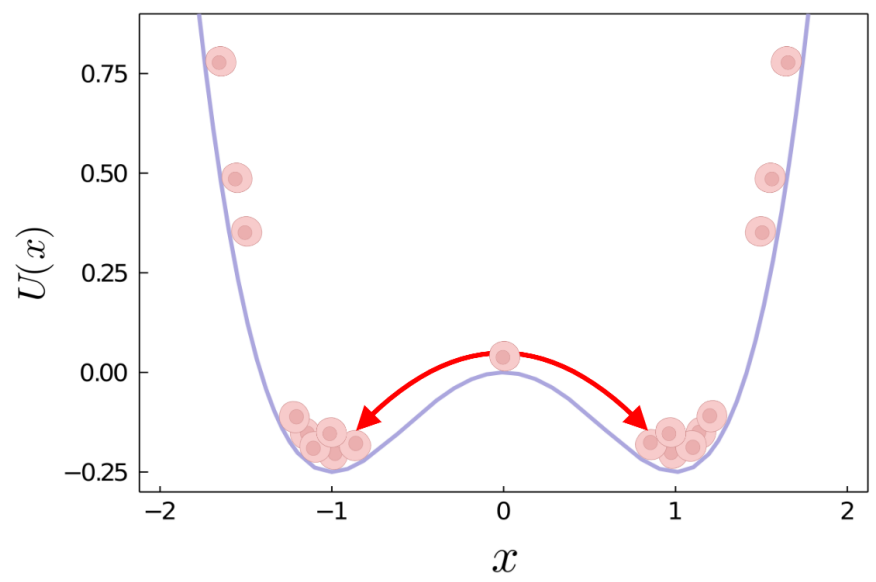

Figure 1: (A) Waddington's classical representation of the epigenetic landscape where cells are described as marbles rolling downhill. The landscape is shaped by the underlying gene regulatory network. Here, we overlay cells navigating the landscape with less differentiated cells starting at the top and traversing downhill. Occasionally, a cell meets a branching point (ii) where it must decide which path to follow. This bifurcation represents a cell-fate decision point. Eventually, differentiated cells come to rest in the valleys of the landscape (outlined in purple) representing areas of low potential and high probability. The system represents a supercritical pitchfork bifurcation and is monostable (i) (stable less differentiated cell state) until it reaches a bifurcation point (ii), where after it becomes bistable (iii) (two distinct stable cell states). (B) Cells moving in the potential function $U(x)$, representing a one-dimensional version of the landscape in (A). The figure in (A) is adapted from [3].

an increment in the Wiener process. Often the noise component $g(x)$ is taken to be independent of the state of the system (i.e. $g(x)$ is constant), and has become known as additive noise. When the noise component $g(x)$ depends on state of the system, the noise is said to be multiplicative [11.

The recent availability of single-cell transcriptomic data has motivated a number of different approximations to potential functions that can serve as mathematical descriptions of the epigenetic landscape [12, 13, 14. Such approximations are often referred to as quasi-potential landscapes. The most popular technique for reconstructing the potential landscape is based upon the steady-state probability distribution, $P_{s}(x)$, of the stochastic system given in $\mathrm{Eq}$ (2) [15, 16, 17, 18, 19]. Here the elevation of the landscape relates to the inverse of the probability density function of the gene expression space. States with the highest probability density, indicated by peaks in the distribution, will have the lowest potential, and thus will correspond to valleys on the landscape. More precisely, the approximated potential landscape is evaluated as [15],

$$
U_{q}(x) \propto-\ln \left(P_{s}(x)\right)
$$

In practise, the steady-state distribution is obtained either by solving (analytically) the associated Fokker-Planck equation (FPE) [20, 21] or, more commonly, by way of simulations of the corresponding SDE [15, 16, 22, 19, 23. To avoid ambiguity, throughout we refer to the estimated landscape $U_{q}(x)$ as the probabilistic landscape, and refer to $U(x)$ as the potential or deterministic landscape.

In this paper, we consider simple one-dimensional stochastic systems to examine the recovery of the potential landscape $U(x)$ by way of the probabilistic landscape $U_{q}(x)$, using Eq $\sqrt{3}$. We demonstrate mathematically that this recovery is strongly dependent on interplay between noise and the deterministic dynamics of the system, as well as highlighting some additional complexities that arise in the presence of biological noise. We further demonstrate some of the limitations to inferring the underlying regulatory dynamics of a noisy system from experimental data. The systems we consider display rich qualitative behaviour, but are simple enough to be analytically tractable.

\section{Noisy gene regulatory networks shape the land- scape}

During differentiation, we often observe increased variability in the expression of specific genes between cells of the same type [24, 25, 26, 27, 28. These changes in transcriptional variability are intrinsically linked to the inherent stochasticity of the transcription and translation processes 29, 30, 31, 32, and appear to be intimately related to differentiation initiation and commitment 33]. As such, the epigenetic landscape, shaped by the underlying gene regulatory network (GRN), is subject to the effects of stochastic fluctuations or noise.

Noise may impact the landscape differently depending on the deterministic and stochastic dynamics of the system. For example, noise may have a purely disorganising effect on the landscape, where the deterministic dynamical structure remains largely intact (i.e. the position and 
bioRxiv preprint doi: https://doi.org/10.1101/2020.12.21.423724; this version posted December 22, 2020. The copyright holder for this

\section{Box 1 | Notation and Terminology}

\begin{tabular}{|c|c|c|}
\hline$x$ & - & Variable denoting the state of the system \\
\hline$f(x)$ & - & Deterministic dynamics of a stochastic dynamical system \\
\hline$g(x) d W t$ & - & Stochastic dynamics of a stochastic dynamical system \\
\hline$d W t$ & - & Increment in the Wiener process \\
\hline$P_{s}(x)$ & - & Steady-state probability distribution of a stochastic dynamical system \\
\hline$U(x)$ & - & Potential function or deterministic landscape \\
\hline$U_{q}(x)$ & - & $\begin{array}{l}\text { Probabilistic landscape computed as logarithm of the inverse of the steady-state } \\
\text { probability distribution of a stochastic system }\end{array}$ \\
\hline$\alpha$ & - & Stochastic integration convention \\
\hline$\sigma$ & - & Noise intensity \\
\hline$\sigma_{c, \alpha}$ & - & $\begin{array}{l}\text { Critical noise (resulting in a qualitative change in the steady-state probability } \\
\text { distribution) for a given stochastic integration convention }\end{array}$ \\
\hline
\end{tabular}

number of valleys and hills on the landscape remain fixed), but cells jostle around the system and occasionally, with an increase in noise intensity, hop from one stable state to another [11. Mathematically, this changes the steadystate distribution $P_{s}(x)$ from a family of delta-peaks to a broader distribution, with mass centered around the family of peaks - often referred to as a "flattening" of the distribution. By way of Eq. (3), this also corresponds to a flattening of the landscape. In addition to these effects, noise may result in qualitative changes to the landscape, by modifying both the position and number of valleys and hills 11. Here the mathematical equivalent is not only a broadening of $P_{s}(x)$, but a shift in positions of the mode(s), as well as the appearance or disappearance of modes. We will see below how noise can drive the creation of new valleys and hills, or destroy existing ones on the deterministic landscape.

Stochastic fluctuations inherent to gene expression arise from both internal and external sources [29, 34, 35, 36, 37. The former originates from probabilistic chemical reactions taking place inside the cell at low concentrations, while the latter refers to stochasticity in the cells everchanging physical and chemical environment. Consequently, the stochastic dynamics of any GRN reflect both the internal and external noise sources at play. Furthermore, noise may enter the system in an additive or multiplicative manner. The GRN may be regulated by the internal state of the cell (e.g. stochastic fluctuations in mRNA and protein levels, feedback loops 38 and higher order interactions and dependencies [39]), as well as the cell's microenvironment [40, 41]. Based on this, it is expected that the stochastic dynamics of the GRN is multiplicative in nature 42]. Mathematically then, noise is no longer a constant term in Eq. (2), but rather a function of the system's state. Moreover, noise may now take on a variety of forms depending on how it relates to the state of the system. When these mathematical subtleties are taken into account, there may be substantial deviations between the deterministic and probabilistic landscapes, which we demonstrate and discuss more formally below. A natural question then arises: what form of noise is relevant for cellular differentiation, and how does it affect the landscape? Here we are guided by a growing body of experimental evidence from single-cell expression analyses, that show that cells undergoing differentiation display an increase in noise intensity at cell-fate decision points. For a recent summary of this phenomenon in different cell types see [43. Indeed, in one of the first articles to mathematically formalise the link between stochasticity and the epigenetic landscape, the authors propose that noise is highest during cell-fate decision points of development 41 .

Taken together, we consider different multiplicative noise forms where the intensity is high at cell-fate decision points, and comparatively lower at differentiated cell states $\sqrt{2}$. We also find it pertinent to consider additional noise forms that will assist in demonstrating the nuances that arise in computing the probabilistic landscape in the presence of stochasticity.

\footnotetext{
${ }^{2}$ We adopt the terminology of [2], and use cell states to describe the transcriptional output of a GRN. Cells progress through a series of cell states to arrive at their eventual cell fate.
} 
bioRxiv preprint doi: https://doi.org/10.1101/2020.12.21.423724; this version posted December 22, 2020. The copyright holder for this preprint (which was not certified by peer review) is the author/funder, who has granted bioRxiv a license to display the preprint in perpetuity. It is made available under aCC-BY 4.0 International license.

\section{Multiplicative noise and reconstructing the poten- tial landscape}

As discussed above, multiplicative noise is the expectation rather than the exception in biological systems. The introduction of a state-dependent noise component, $g(x)$, introduces a number of subtleties in using the distributionbased method given in Eq. (3) for reconstructing the potential landscape. While some of these complications are widely understood in physics [11, 44], they are perhaps less so in a biological context.

The first subtlety arises in solving the SDE in Eq. (2). The integration of Eq. (2) now involves stochastic integrals, which can be interpreted according to different conventions. Such integrals are calculated in the standard way via discretisation

$$
\int_{0}^{t} g\left(x_{t}\right) d W_{t}=\lim _{n \rightarrow \infty} \sum_{k=1}^{n} g\left(x_{t_{k}^{*}}\right)\left(W\left(t_{k}\right)-W\left(t_{k-1}\right)\right),
$$

for increasingly finer partitions $0=t_{0}<t_{1}<\cdots<t_{n}-$ $1<t_{n}=t$. Unlike standard integration however, the integral now depends on the point $t_{k}^{*}=t_{k-1}+\alpha\left(t_{k}-\right.$ $\left.t_{k-1}\right)$ in $\left[t_{k-1}, t_{k}\right]$ where the process $W(t)$ is evaluated. Here $\alpha \in[0,1]$ and is known as the stochastic integration convention. The two most common conventions are the Itô interpretation, where $t_{k}^{*}=t_{k-1}$ (that is, $\alpha=0$ ), and the Stratonovich interpretation, where $t_{k}^{*}=\left(t_{k}+t_{k-1}\right) / 2$ (that is, $\alpha=0.5$ ). Allowing for this, it is possible to show that the SDE in Eq. (2) is more accurately presented as

$$
d x=\left(f(x)+\alpha g(x) \frac{\partial g(x)}{\partial x}\right) d t+g(x) d W_{t} .
$$

The presence of multiplicative noise can then be seen to introduce an additional term, $\alpha g(x) \frac{\partial g(x)}{\partial x}$, known as the noise-induced drift term [11. The dependence of Eq. (5) on the stochastic integration convention $\alpha$ is also reflected in the steady-state probability distribution $P_{s}(x)$ of the state of the system $x$, and consequently, the probabilistic landscape $U_{q}(x)$ : explicitly, it is known that

$$
P_{s}(x)=\frac{\mathcal{N}}{g^{2}(x)} \exp \left(2 \int^{x} \frac{f(z)+\alpha g(z) g^{\prime}(z)}{g^{2}(z)} d z\right),
$$

where $\mathcal{N}$ is the normalisation constant. The probabilistic landscape as computed by Eq. (3) is then given by

$$
U_{q}(x)=\ln \left(\frac{g^{2}(x)}{\mathcal{N}}\right)-2 \int^{x} \frac{f(z)+\alpha g(z) g^{\prime}(z)}{g^{2}(z)} d z .
$$

While both the Itô and Stratonovich interpretations are legitimate, Eq.'s (6) and (7) show that different choices of $\alpha$ can lead to different solutions for $P_{s}(x)$, and subsequently, $U_{q}(x)$. Thus, from a modelling perspective, an SDE is somewhat meaningless until it is assigned a stochastic integration convention. Deciding which interpretation should be adopted for a given SDE, known as the ItôStratonovich dilemma, has been widely debated and remains a source of controversy even today 45]; for more information refer to Box 2. To complicate matters even further, it is possible that the stochastic integration convention required to correctly interpret an SDE may vary as the parameters of the system change 46.

A second subtlety arises in the computation of the probabilistic landscape, $U_{q}(x)$. In the case of additive noise, it is clear from Eq. (7), that there is no contribution from the noise-induced drift term, and so the deterministic potential will be recovered as $U_{q}(x)$, up to a scale and shift; this is implicit in the original justification for computing the landscape as $U_{q}(x)$ [15]; see also [13, 47]. In the case of multiplicative noise, however, even for a fixed stochastic integration convention $\alpha$, the closeness of the probabilistic landscape $U_{q}(x)$ to the deterministic landscape $U(x)$, will strongly and non-trivially depend on the interaction between the deterministic and stochastic dynamics.

In the following section, we use a set of simple illustrative examples to demonstrate the dependence of $U_{\mathrm{q}}(x)$ on the choice of the stochastic integration convention $\alpha$, as well as the functions $f(x)$ and $g(x)$. In particular, we demonstrate and prove mathematically that for common choices of $\alpha$, the probabilistic landscape of a bistable system (consisting of two stable fixed points) may in fact become monostable (consisting of a single fixed point) in the presence of multiplicative noise. Thus, unlike in the case where the noise component $g(x)$ is constant, the probabilistic landscape will not necessarily reflect the deterministic potential landscape.

A further important consideration is the identifiability of the underlying deterministic dynamics in the presence of stochasticity. In the context of Eq. (6), this corresponds to asking whether or not $f(x)$ is uniquely determined by $U_{q}(x)$. We provide an example that shows that there are systems characterised by strikingly different $f(x)$ and different $g(x)$ that give rise to the same steady-state probability distribution, and by extension, the same probabilistic landscape. Thus, it is not possible to identify the deterministic dynamics on the basis of the observed landscape $U_{q}(x)$ (or single-cell snapshot data) alone. Despite this, we show that for one-dimensional stochastic systems, any one of $g(x), f(x)$ and $P_{s}(x)$ can be uniquely determined from knowledge of the other two. Consequently, the deterministic dynamics $f(x)$ (equivalently, $U(x)$ by way of Eq. (1)), can be determined from $U_{q}(x)$, only once we obtain additional information about the noise component $g(x)$.

\section{Effect of noise form and intensity on the landscape}

\section{A double-well potential model}

The model we consider here is not intended to represent any explicit biological system, but rather serves as an illustration [48] for studying the impact of noise on the stationary solutions of stochastic differential equations, and the corresponding probabilistic landscapes. While the model may be simple, it is able to give rise to intricate and biologically relevant behaviour such as bifurcations; these 
bioRxiv preprint doi: https://doi.org/10.1101/2020.12.21.423724; this version posted December 22, 2020. The copyright holder for this preprint (which was not certified by peer review) is the author/funder, who has granted bioRxiv a license to display the preprint in perpetuity. It is made available under aCC-BY 4.0 International license.

\section{Box 2 | The Itô-Stratonovich Dilemma}

The source of controversy

Stochastic differential equations (SDEs) are frequently used to model real-world systems subject to random fluctuations. These fluctuations are often mathematically represented as white noise, also referred to as a Wiener process or Brownian motion. When the noise component is state-dependent (multiplicative), the resulting SDE (see Eq. 22) is no longer well-defined without additional information: the precise behaviour depends on the adopted convention for stochastic integration. Essentially, the difficulties arise in solving the SDE and attempting to evaluate stochastic integrals of the form $\int_{0}^{T} g\left(x_{t}\right) d W_{t}$. In the standard Riemann integral of integral calculus, we consider increasingly finer partitions $0=t_{0}<t_{1}<\cdots<t_{n}=T$ of the interval $[0, T]$ over which we wish to integrate. For each sub-interval $\left[t_{k-1}, t_{k}\right]$ in a partition of $[0, T]$, the integral is approximated by a rectangle of height given by a value of the integrand $g(x, t)$ at some point $t_{k}^{*}$ in $\left[t_{k-1}, t_{k}\right]$. The overall integral is then approximated by the sum of these rectangles - the so-called Riemann sum. Conveniently, for a smooth function, the limit over increasingly finer partitions does not depend on the precise partitions (provided the subinterval lengths go to 0 ) nor on the precise evaluation of $g(x, t)$ within the intervals, so that the integral itself can be defined as the limit. For stochastic processes this is no longer true: different choices of the evaluation point within the sub-intervals can give a different outcome. Mathematically, we can express this dependence as $t_{k}^{*}=t_{k-1}+\alpha\left(t_{k}-t_{k-1}\right)$, where the parameter $\alpha$ is from $[0,1]$ and is known as the stochastic integration convention. Common choices include $\alpha=0$ or $\alpha=0.5$, known as the Itô or Stratonovich interpretation, respectively.

\section{Common stochastic calculus prescriptions}

Rewriting an SDE, in conjunction with a stochastic convention $\alpha$, gives Eq. (5) - and it is exactly the value of $\alpha$ that is at the heart of the Itô-Stratonovich debate. The Itô interpretation evaluates the integrand for the $k^{\text {th }}$ interval $\left[t_{k-1}, t_{k}\right]$ at $t_{k-1}$. Thus the value at $t_{k-1}$ is chosen based only on information available at that time; this is often referred to as non-anticipating, and means that the Itô integral is a martingale; a useful mathematical property [10]. A consequence is that there is no correlation between $g\left(x_{t}\right)$ and $d W t$. In contrast, the Stratonovich interpretation evaluates the integral at $\frac{t_{k-1}+t_{k}}{2}$, meaning that the value taken depends on the future time $t_{k}$; it is said to be anticipating. This results in correlation between $g\left(x_{t}\right)$ and $d W t$.

\section{Mathematical equivalence}

For a fixed SDE, the Itô and Stratonovich interpretations yield different results. Once an initial prescription is assigned to the SDE however, one can easily convert between the two interpretations, either by adding or subtracting the noise-induced drift term [10, 49] (see main text). Thus, for every Itô-SDE there is a StratonovichSDE with an identical solution. If we wish to model a known system subject to stochasticity in order to predict the behaviour, we are faced with the dilemma of which stochastic calculus is the appropriate one, given they may give different answers. Conversely, if we have observed behaviour and wish to infer the underlying dynamics of the system, then the conundrum again arises, as different equations are required to achieve the given solution depending on the calculus.

\section{Itô or Stratonovich?}

The appropriate stochastic calculus to prescribe in a real-world setting is usually chosen on physical grounds and rests upon the characteristics of the noise. There are no theoretical a priori reasons to recognise one interpretation over the other, however, we may consider the following guidelines:

Itô Stratonovich

Use if the SDE is a continuous approximation to an Use in continuous systems where the SDE represents underlying discrete process the white noise limit of a system subject to real noise.

\begin{tabular}{ll}
\hline Non-anticipating (martingale property) & Anticipating \\
\hline Requires "new" calculus rules i.e. Itô's lemma & Maintains traditional rules of calculus e.g. chain rule \\
\hline Often used in: finance; population evolution & Often used in: physics; engineering; physical sciences \\
\hline
\end{tabular}


are an important hallmark of cell-fate decision making and mark the qualitative changes in cellular phenotype 2]. Examining how noise changes the topology ${ }^{3}$ of the landscape, specifically in the presence of such bifurcation points and lineage branching events, then ultimately provides insights into how it may influence cell-fate decision making. Throughout, we consider the classic case of a single particle moving in a double-well potential, and examine the effects of both a state-independent (additive) and state-dependent (multiplicative) noise component. We define the system in terms of the potential function $U(x)$ described by,

$$
U(x)=\frac{x^{4}}{4}-\frac{x^{2}}{2},
$$

where $x$ represents the state of the system i.e. the entire GRN that shapes the respective landscape. Moving $x$ in state space represents a change in the gene expression profile of a cell. This one-dimensional potential is plotted in Fig. 1(B) and is reminiscent of the classic Waddington landscape, displayed in Fig. 1(A). Here the valleys that represent distinct cell states are depicted by the stable stationary points of the system, occurring at $x_{\min }= \pm 1$, while the hill between them is depicted by the unstable stationary point at $x_{\max }=0$, and represents a transition or intermediate cell state [19, 50.

\section{Additive noise}

We consider first the case of additive noise. Here $g(x)=\sigma$, with noise intensity $\sigma>0$. To build intuition, we begin by considering informally the effects of this noise on the potential landscape defined in Eq. 8). In Fig. 2 , we plot both the potential function $U(x)$ and the noise function $g(x)=1$; these are shown as the purple and red curves respectively. We indicate with blue arrows the net propensity of the system's trajectory, which we define as the net effect of noise on the potential landscape. The most probable regions of the system's trajectory are indicated by the pink cells. As the noise is uniform across the deterministic landscape $U(x)$, the net propensity of the system is proportional to the derivative of the potential landscape $U(x)$. In particular, the net propensity is zero at the stationary points of $U(x)$, and so these will be unaltered by the effects of noise. An increase in noise intensity has only a disorganising effect on the landscape i.e., it merely jostles the cells around in the potential, thereby increasing the probability of a cell to hop from one stable state to another. We now consider the system more formally.

As discussed above, both the Itô and Stratonovich interpretations of the stochastic system lead to the same stationary probability distribution. Thus, the estimated landscape $U_{q}(x)$ will not depend on $\alpha$ when $g(x)$ is constant.

${ }^{3}$ We adopt the terminology of 2, and use topology to describe key features of the landscape such as the number and position of fixed points.
As $f(x)$ is related to $U(x)$ by way of Eq. (1), it follows from Eq.'s (6) and (7), that the stationary probability distribution, and subsequently the probabilistic landscape $U_{q}(x)$, are given by

$$
P_{s}(x)=\frac{\mathcal{N}}{\sigma^{2}} \exp \left(\frac{-2 U(x)}{\sigma^{2}}\right)
$$

and

$$
U_{q}(x)=\frac{2 U(x)}{\sigma^{2}}+\ln \left(\frac{\sigma^{2}}{\mathcal{N}}\right),
$$

respectively. Here $\mathcal{N}$ is the normalisation constant. We display the steady-state probability distribution $P_{s}(x)$ in Fig. 2(A), and the corresponding probabilistic landscape $U_{q}(x)$ in Fig. 2(B). In each figure, the three curves are computed for increasing values of noise $\sigma$. We see that as $\sigma$ increases, both $P_{s}(x)$ and $U_{q}(x)$ flatten, but the maxima of $P_{s}(x)$ and therefore minima of $U_{q}(x)$ remain fixed at $x_{\max }= \pm 1$ and $x_{\min }= \pm 1$, respectively. Consequently, the overall topology of the landscape is preserved in the case of additive noise. That is to say the stable stationary points of the probabilistic landscape $U_{q}(x)$ directly correspond to those of the deterministic landscape or potential, $U(x)$. Note that this does not depend on the specific choices of $f(x)$ and $g(x)$ that we have made here; $U(x)$ will always be recovered as $U_{q}(x)$, albeit scaled and shifted by the noise parameter $\sigma$.

\section{Multiplicative noise}

For multiplicative noise the probabilistic landscape $U_{q}(x)$ is profoundly shaped by the interaction between the deterministic dynamics $f(x)$ (or equivalently $U(x)$ ) and the stochastic dynamics $g(x)$. We begin by considering the effects of different noise forms on our model, showing that the topological features of the deterministic landscape can be distorted by multiplicative noise. Following this, we provide a general result, which shows the fixed points of the deterministic landscape $U(x)$ will be always be preserved by $U_{q}(x)$, when the noise is related to the deterministic landscape by way of a power relationship. A particularly natural way in which noise might arise is when the noise component $g(x)$ is proportional to the deterministic landscape itself. This corresponds to a special case of our general result, and we discuss how the effects of this noise on the landscape are then qualitatively indistinguishable from that of additive noise. Our result holds for any potential function $U(x)$; it is not limited to any specific form such as the double-well potential considered here.

\section{Example 1: Shifting the fixed points}

We begin by considering the effect of the noise component $g(x)=\frac{\sigma}{4}\left(x^{4}-4 x^{2}+8\right)$, for $\sigma>0$, on the model $U(x)$ defined in Eq. (8). In order to build intuition, we display in Fig. 3(A) both the potential function $U(x)$ and noise function $g(x)$ (for $\sigma=1$ ); these are the purple and red curves, respectively. We see that the noise function 

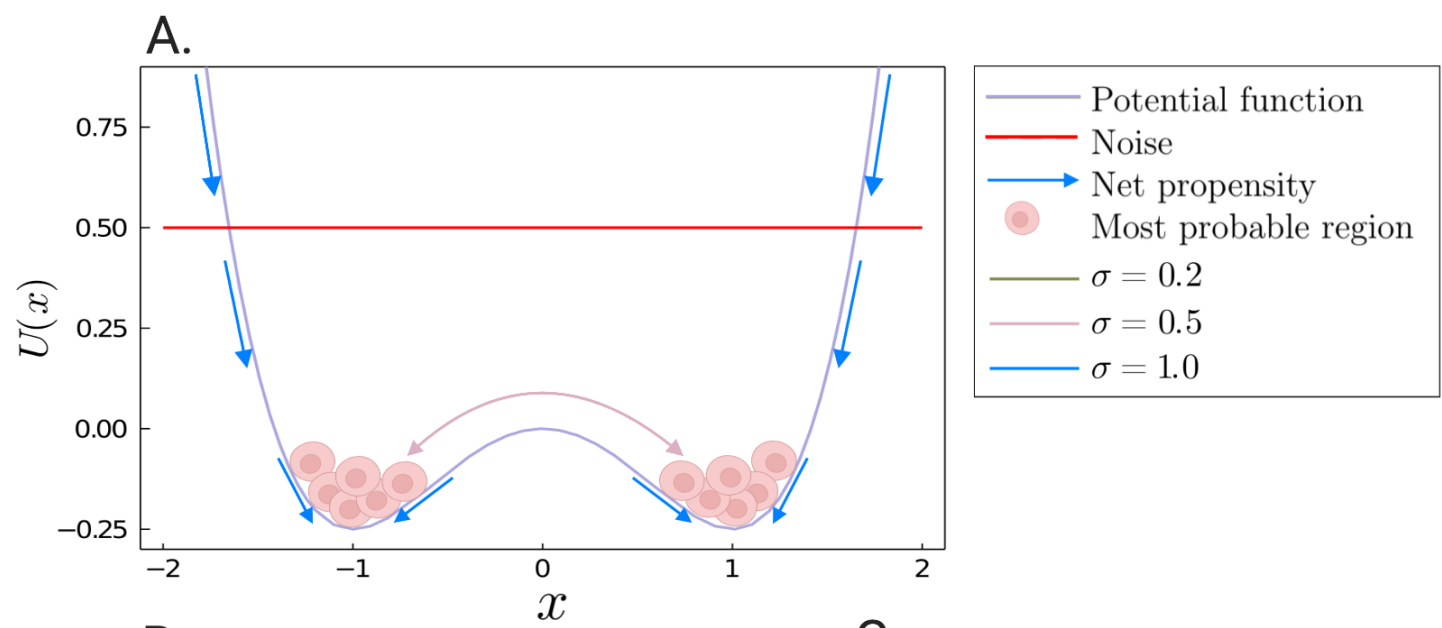

B.
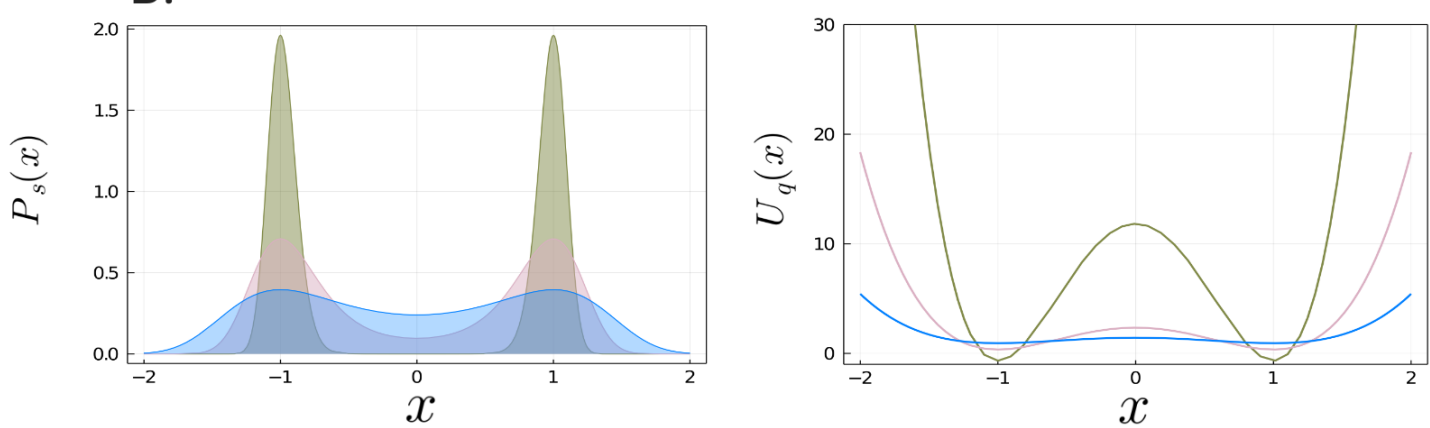

Figure 2: (A) A schematic of the system's trajectory. We consider a deterministic double-well potential (purple curve) driven by additive noise (red curve). The blue arrows represent the net propensity of the system, with cells gathering in the most probable regions. The pink arrow indicates rare transitions from one stable state of the system to the other. (B) Analytical steady-state probability distribution $P_{s}(x)$ given by Eq. 9. (C) The corresponding probabilistic landscape $U_{q}(x)$ given by Eq. 10. Both figures are plotted for the model defined in Eq. 8) with increasing strengths of additive noise. The different colours represent the different levels of noise: the green curve is for $\sigma=0.2$, the pink curve for $\sigma=0.5$ and the blue curve for $\sigma=1.0$. As $\sigma$ increases both $P_{s}(x)$ and $U_{q}(x)$ flatten, making rare transitions between states more probable. The maxima of $P_{s}(x)$ and minima of $U_{q}(x)$ are preserved at $x= \pm 1$.

broadly follows the shape of the potential function, however the stable stationary points of $g(x)$ do not agree with those of $U(x)$ : for $g(x)$ these occur at $x_{\min }= \pm \sqrt{2}$, while for $U(x)$ these occur at \pm 1 . The net propensity of the system is indicated by blue arrows, and the most probable regions of the system's trajectory are represented by the pink cells. As the noise is much higher at the unstable stationary point $\left(x_{\min }=0\right)$ relative to the strength of noise to the left and right of it, cells are pushed away from the unstable point. The net propensity of the system is therefore non-negligible at the stable stationary points of $U(x)$, and so the system's trajectory is more often found in the region slightly beyond the stable stationary points of $U(x)$. The resulting stationary distribution is therefore bimodal, with modes occurring beyond $x_{\max }= \pm 1$. We now formalise this intuition.

As the noise function $g(x)$ is state-dependant, the stochastic system may be interpreted in either the Itô or Stratonovich sense, leading to different solutions for the steady-state probability distribution $P_{s}(x)$ and, subsequently, the probabilistic landscape $U_{q}(x)$. The dependence on the stochastic integration convention $\alpha$ can be seen from Eq.'s (6) and (7) with $f(x)=x-x^{3}$ and $g(x)=\frac{\sigma}{4}\left(x^{4}-4 x^{2}+8\right)$; explicitly we have

$$
\begin{aligned}
P_{s}(x) & =\frac{16 \mathcal{N}}{\sigma^{2}} \exp \left(2(\alpha-1) \ln \left(x^{4}-4 x^{2}+8\right)\right. \\
+ & \left.\frac{1}{\sigma^{2}}\left(\tan ^{-1}\left(1-\frac{x^{2}}{2}\right)-\frac{2\left(x^{2}-6\right)}{x^{4}-4 x^{2}+8}\right)+C\right)
\end{aligned}
$$

and

$$
\begin{aligned}
U_{q}(x) & =\ln \left(\frac{\sigma^{2}}{16 \mathcal{N}}\left(x^{4}-x^{2}+8\right)^{2(1-\alpha)}\right) \\
& -\frac{1}{\sigma^{2}}\left(\tan ^{-1}\left(1-\frac{z^{2}}{2}\right)-\frac{2\left(z^{2}-6\right)}{z^{4}-z^{2}+8}\right)-C,
\end{aligned}
$$

where $\mathcal{N}$ is the normalisation constant and $C$ is the constant of integration.

In Fig. 3. we plot the steady-state probability distribution $P_{s}(x)$ and the corresponding landscape $U_{q}(x)$, for both of the Itô $(\alpha=0)$ and Stratonovich $(\alpha=0.5)$ interpretations. We use solid lines to represent the Itô interpretation (Fig. 3(B\&D)), while dashed lines represent the Stratonovich interpretation (Fig. 3(C\&E)). Each figure is 

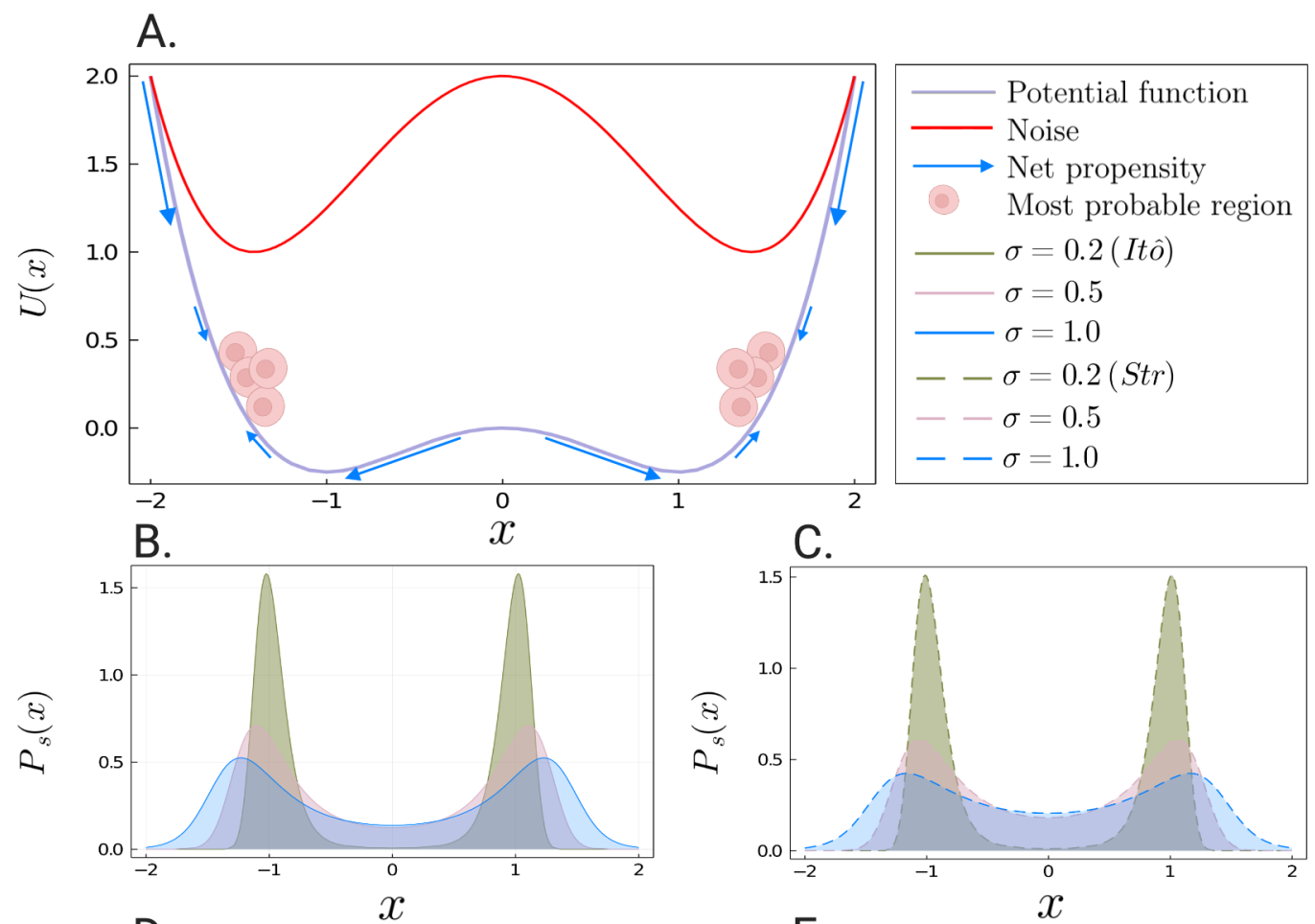

C.
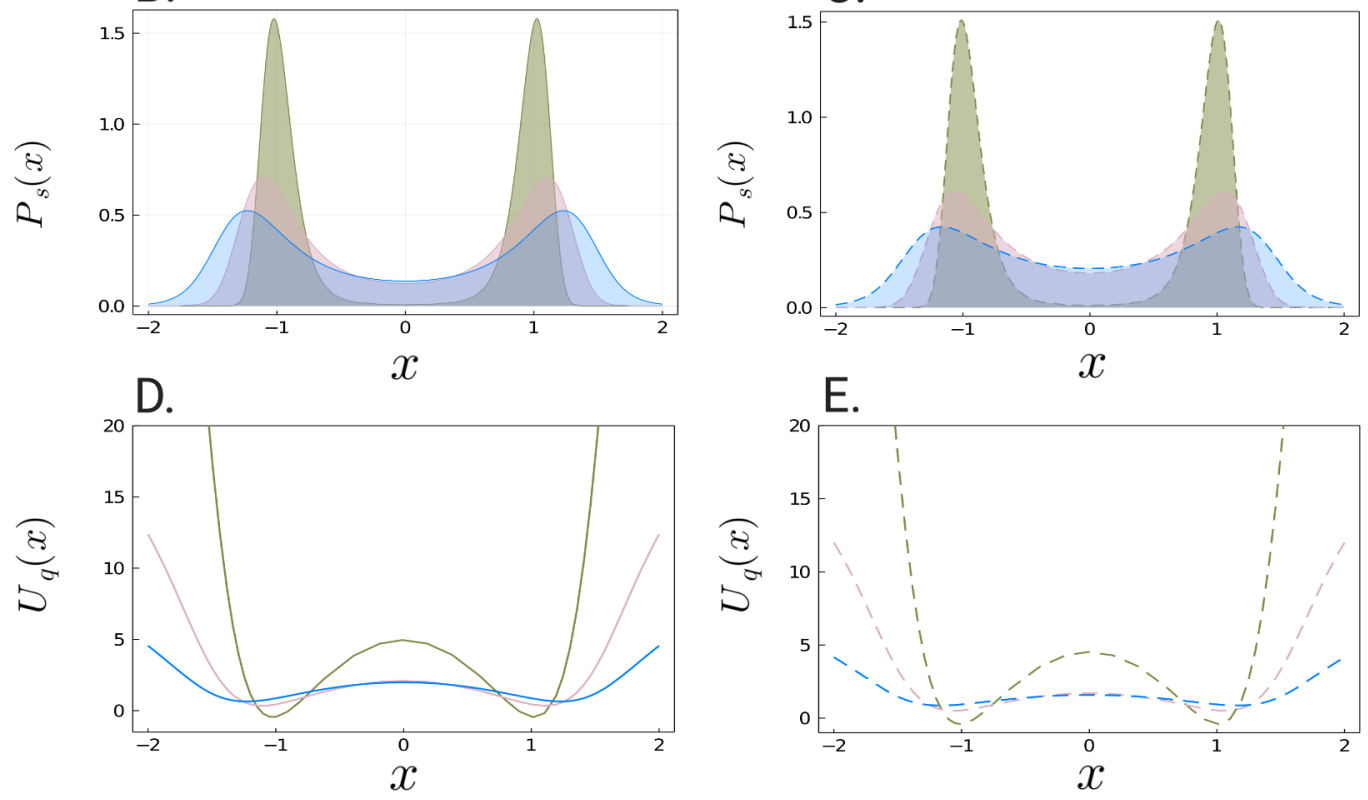

E.

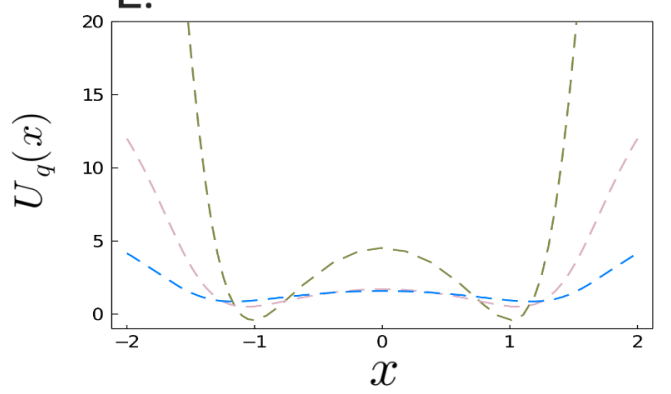

Figure 3: (A) A schematic of the system's trajectory. Here we consider a deterministic double-well potential driven by multiplicative noise. The net propensity of the system (as represented by the blue arrows) is to drive cells away from the origin. Cells are then more often found beyond the stationary points of the deterministic landscape $U(x)$. (B\&C) Analytical steady-state probability distribution $P_{s}(x)$ given by Eq. [11, for $\alpha=0$ (solid lines) and $\alpha=0.5$ (dashed lines), respectively. (D\&E) The corresponding landscape $U_{q}(x)$ given by Eq. 12 , for $\alpha=0$ (solid lines) and $\alpha=0.5$ (dashed lines), respectively. Figures (B-E) are plotted for the model defined in Eq. 8 ) with increasing strengths of multiplicative noise. Here $\sigma=0.2$ is represented as the green curve, $\sigma=0.5$ as the pink curve, and $\sigma=1.0$ as the blue curve. As $\sigma$ increases, the maxima of $P_{s}(x)$ and (resp. minima of $U_{q}(x)$ ) are shifted away from the origin towards the fixed points of the noise curve $g(x)$. As $\sigma$ increases both $P_{s}(x)$ and $U_{q}(x)$ flatten.

plotted for increasing strengths of multiplicative noise. As with additive noise, an increase in $\sigma$ broadens the steadystate probability distribution $P_{s}(x)$, leading to a decrease in the depth of the wells of the corresponding landscape $U_{q}(x)$. The modes of the distribution (resp. wells of the landscape) however do not remain fixed: they are now shifted away from the origin. This displacement in the modes (wells) occurs for both of the stochastic interpretations. In the limit of large noise (i.e. $\sigma \rightarrow \infty$ ), the modes of $P_{s}(x)$ (wells of $U_{q}(x)$ ) approach the stable stationary points of the noise function $g(x)$, which occur at $x_{\min }= \pm \sqrt{2}$.

While the effects of the noise $g(x)$ on the deterministic potential are visually almost indistinguishable for the Itô
(Fig. 3(B\&D)) and Stratonovich (Fig. 3(C\&E))) interpretations of the stochastic system, under close inspection it can be seen that the Stratonovich interpretation has produced slightly flatter curves. In fact, they are also slightly narrower. For any given noise intensity $\sigma$, the modes of $P_{s}(x)$ (resp. wells of $U_{q}(x)$ ) are shifted further away from the origin under the Itô interpretation, in comparison to the Stratonovich interpretation. The broadening of $P_{s}(x)$ (resp. flattening of $U_{q}(x)$ ) however, is more pronounced under the Stratonovich interpretation, for any given noise intensity $\sigma$.

A similar example can be constructed using the noise component $g(x)=\frac{\sigma}{8}\left(2 x^{4}-x^{2}+16\right)$. Again, this noise curve broadly follows the shape of the potential landscape 
$U(x)$, but has minima occuring at $x_{\min }= \pm \frac{1}{2}$. This noise now shifts the stationary points of the landscape towards the unstable stationary point at the origin, which approach $\pm \frac{1}{2}$ in the limit of large noise. Details of this example can be found in the supplementary material.

\section{Example 2: Creation and destruction of fixed points}

We next consider the effect of the noise component $g(x)=\sigma\left(1+x^{2}\right)$, for $\sigma>0$ on the double-well potential $U(x)$. We begin by considering informally the effects of this noise on $U(x)$. In Fig. 4(A), we plot both $U(x)$ and the noise function $g(x)$ as the purple and red curves, respectively. As the noise is much higher at the left-and rightmost extremes of the landscape, relative to the strength of noise at the origin, cells are pushed towards the unstable stationary point of $U(x)$. The net propensity of the system (indicated by the blue arrows) is non-negligible at the stable stationary points of $U(x)\left(x_{\min }= \pm 1\right)$, and so the system's trajectory is more often found either side of the unstable point, now closer to the origin. For lower noise intensities, the resulting steady-state probability distribution will therefore be bimodal, with modes occurring between $(-1,1)$. When the noise intensity becomes large enough, eventually the two modes (corresponding to the most probable regions of cells) will collide at the origin, resulting in a unimodal distribution.

Again, as the noise is state-dependant, the stochastic system may be interpreted in either the Itô or Stratonovich sense. Thus, the steady-state distibution $P_{s}(x)$ and, therefore, the probabilistic landscape $U_{q}(x)$ will depend on $\alpha$. To see this explicitly, we consider Eq.'s (6) and (7) with $f(x)=x-x^{3}$ and $g(x)=\sigma\left(1+x^{2}\right)$; we have

$$
\begin{array}{r}
P_{s}(x)=\frac{\mathcal{N}}{\sigma^{2}\left(1+x^{2}\right)^{2}} \exp \left(\frac { 1 } { \sigma ^ { 2 } } \left(\left(2 \alpha \sigma^{2}-1\right) \ln \left(1+x^{2}\right)\right.\right. \\
\left.\left.-\frac{2}{\left(1+x^{2}\right)}+C\right)\right)
\end{array}
$$

and

$$
\begin{aligned}
& U_{q}(x)=\ln \left(\frac{\sigma^{2}\left(1+x^{2}\right)^{2}}{\mathcal{N}}\right) \\
& -\frac{1}{\sigma^{2}}\left(\left(2 \alpha \sigma^{2}-1\right) \ln \left(1+x^{2}\right)-\frac{2}{\left(1+x^{2}\right)}+C\right),
\end{aligned}
$$

where $\mathcal{N}$ is the normalisation constant and $C$ is the constant of integration.

In Fig. 4 we plot the steady-state probability distribution $P_{s}(x)$ and the corresponding landscape $U_{q}(x)$, for both stochastic integration conventions $\alpha=0$ (Fig. 4(B\&D) and $\alpha=0.5$ ((Fig. 4(C\&E)). Again, each figure is plotted for the model in Eq. (8), with increasing strengths of multiplicative noise. Solid lines represent the Itô interpretation $(\alpha=0)$, and dashed lines represent the Stratonovich interpretation $(\alpha=0.5)$. As the noise intensity $\sigma$ increases, we see a broadening in the steady-state probability distribution $P_{s}(x)$, leading to a decrease in the depth of the wells of the corresponding landscape $U_{q}(x)$. The shape of the probabilistic landscape $U_{q}(x)$, however has a major departure from that of the potential $U(x)$. More specifically, the modes of the steady-state probability distribution, and subsequently the minima of the probabilistic landscape are displaced towards the origin, as the noise intensity $\sigma$ increases. The extent of these effects vary according to stochastic integration convention $(\alpha=0$ or $\alpha=0.5)$. This can be seen explicitly from the minima of the probabilistic landscape $U_{q}(x)$, which are given by

$$
x_{\min }= \pm \sqrt{\frac{1-2 \sigma^{2}(1-\alpha)}{1+2 \sigma^{2}(1-\alpha)}},
$$

Unlike in the case of additive noise, the minima of $U_{q}(x)$ agree with those of the potential landscape $U(x)$ only in the limit as $\sigma$ approaches 0 (i.e. the weak noise limit). While for $\sigma \geq 1 / \sqrt{2(1-\alpha)}$, the stable stationary points $\left(x_{\text {min }}\right)$ of $U_{q}(x)$ collide, resulting in a single stable stationary point at the origin. We refer to the point

$$
\sigma_{c, \alpha}=\frac{1}{\sqrt{2(1-\alpha)}}
$$

at which the system transitions from bistable to monstable as the critical noise. Notably, this point depends on the stochastic interpretation $\alpha$. For the Itô interpretation, the critical noise $\sigma_{c, 0}=\frac{1}{\sqrt{2}}$, while for the Stratonovich interpretation $\sigma_{c, 0.5}=1$. Thus, under the Stratonovich interpretation, a higher noise intensity is required to drive the system from bistable to monstable. The discrepancy between the stochastic interpretations can also be seen in Fig. 5. where $U_{q}(x)$ is plotted for both interpretations with noise intensity $\sigma=\sigma_{c, 0}=\frac{1}{\sqrt{2}}$.

In this example, we have demonstrated that the probabilistic landscape of a bistable system may in fact become monostable in the presence of multiplicative noise. In other words, multiplicative noise can destroy the fixed points of a system. In the next section, we will see how multiplicative noise can also drive the creation of fixed points: a monostable system under the influence of mulitplicative noise can become bistable. It is precisely these observations that have important consequences for dynamical systems undergoing a bifurcation in the presence of multiplicative noise. Specifically, the stochastic system $U_{q}(x)$ may display qualitatively different behaviour from the deterministic system $U(x)$. We discuss bifurcations of stochastic dynamical systems in more detail further on.

\section{Preservation of fixed points under multiplicative noise}

Let $U(x)$ be any one-dimensional potential function, and assume that $g(x)$ is related to $U(x)$ by way of the following power relationship,

$$
g(x)=\sigma(U(x)+C)^{\delta} .
$$

Here $\sigma>0$ is the noise intensity and $C, \delta$ are any nonnegative real numbers. Further assume that $U(x)+C>0$, 

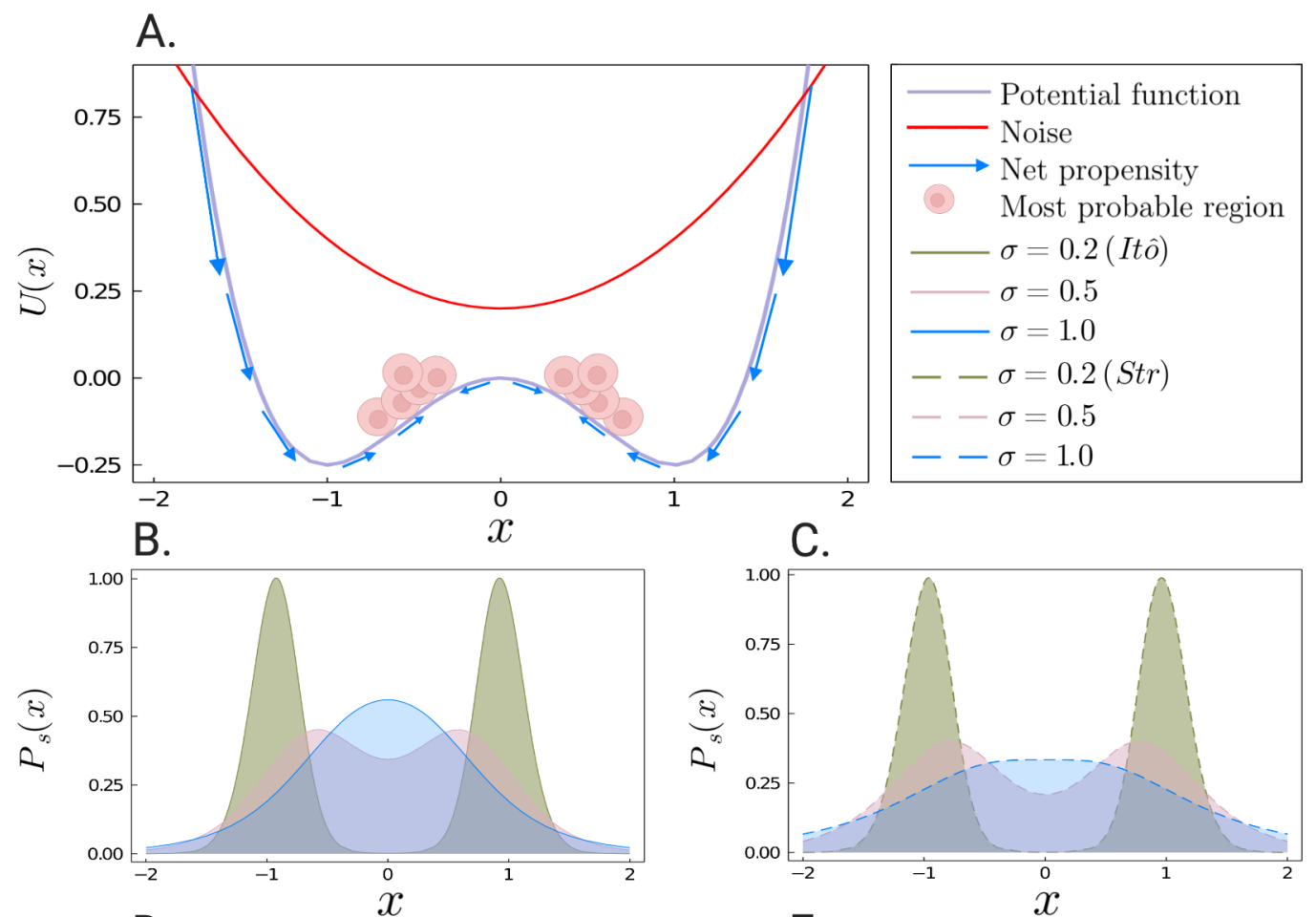

D.

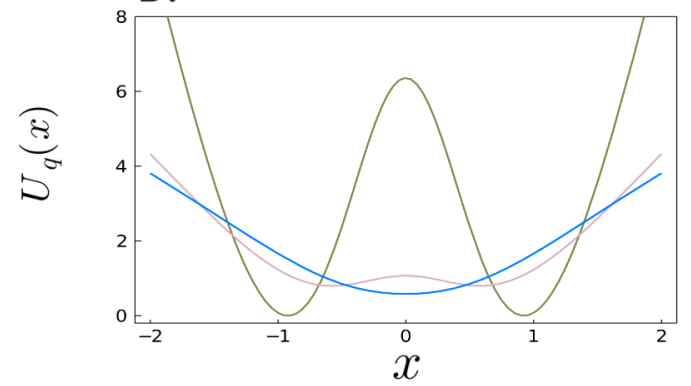

E.

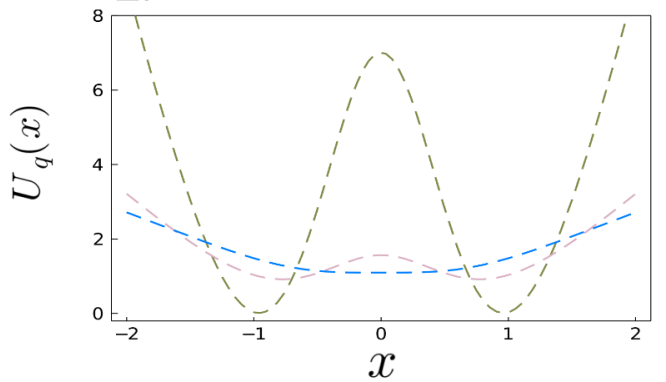

Figure 4: (A) A schematic of the system's trajectory. We consider a deterministic double-well potential driven by multiplicative noise. The net propensity (blue arrows) pushes cells toward the origin. (B\&C) Analytical steady-state probability distribution $P_{s}(x)$ given by Eq. 13 , for $\alpha=0$ (solid lines) and $\alpha=0.5$ (dashed lines), respectively. (D\&E) The corresponding landscape $U_{q}(x)$ given by Eq. 14 ), for $\alpha=0$ (solid lines) and $\alpha=0.5$ (dashed lines), respectively. Figures (B-C) are plotted for the model defined in Eq. 8 with increasing strengths of multiplicative noise. Here $\sigma=0.2$ is represented by the green curve, $\sigma=0.5$ by the pink curve, and $\sigma=1.0$ by the blue curve. As $\sigma$ increases, the maxima of $P_{s}(x)$ and minima of $U_{q}(x)$ are shifted towards the origin until eventually they collide (blue curve). As $\sigma$ increases both $P_{s}(x)$ and $U_{q}(x)$ flatten.

for all $x$, so that $g(x)$ is strictly positive. Then it can be shown that, for any stochastic integration convention $\alpha$, the fixed points of the approximated landscape $U_{q}(x)$ correspond precisely to those of $U(x)$ (when they exist). Thus, for multiplicative noise of the form given in Eq. (17), the topological structure of $U(x)$ is preserved by the probabilistic landscape $U_{q}(x)$, in a similar way to that of additive noise. Indeed, additive noise is a particular case of Eq (17), corresponding to when $n=0$. The proof of this general result is provided in the supplementary material.

A natural noise form that satisfies the broader features of noise observed in cellular differentiation arises when $g(x)$ is proportional to the deterministic landscape itself. In the context of Eq. (17), this corresponds to the particular case where $C=0$ and $\delta=1$. An example demonstrat- ing the effect of this noise on the model defined in Eq. (8) can be found in the supplementary material; the effects on the stationary distribution and probabilistic landscape can be seen to be qualitatively identical to that of additive noise.

\section{Identifiability and limitations to inferring dynam- ics}

As discussed above, a stochastic dynamical system is in general characterised by a stochastic integration convention $\alpha$, in unison with a deterministic component $f(x)$ and stochastic component $g(x)$. In practise however, it is rarely the case that these components are known: experimental data is of the form $P_{s}(x)$, which provides only a 
A.

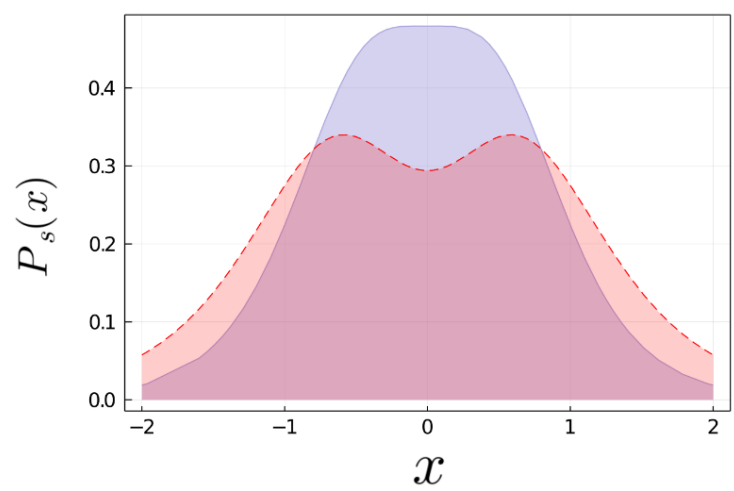

B.

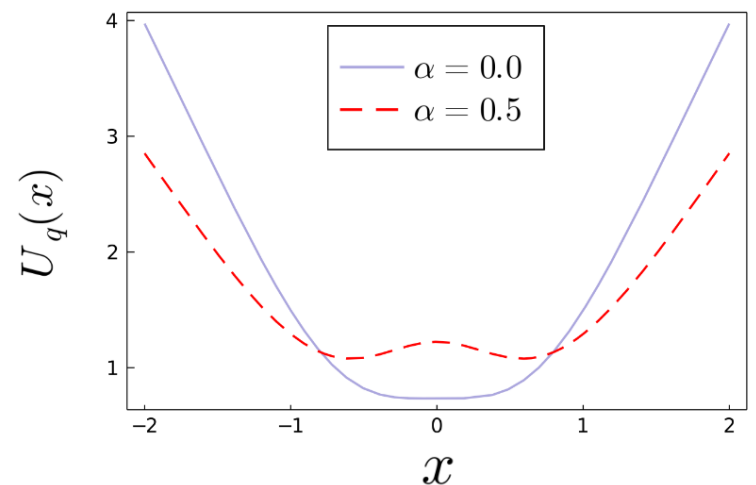

Figure 5: (A) Analytical steady-state probability distribution $P_{s}(x)$ given by Eq. 13 and (B) the corresponding landscape $U_{q}(x)$ given by Eq. (14), plotted for a fixed noise intensity $\sigma=0.7$. The solid line is in the Itô interpretation and the dashed line in the Stratonovich interpretation. The displacement of the maxima of $P_{s}(x)$, and subsequently the minima of $U_{q}(x)$, depend on the prescribed calculus. At $\sigma=0.7$ the Itô interpretation is monostable while the Stratonovich interpretation is still bistable. Both prescriptions give rise to a drift when compared with the deterministic dynamics, although the drifts are weighted differently.

static picture of the long-term, average dynamics of the system. Thus, some of the dynamical information of the system is lost in $P_{s}(x)$. How much then can we deduce of the underlying stochastic dynamics $(f(x)$ and $g(x))$, as well as the stochastic calculus $\alpha$, from the measured stationary distribution $P_{s}(x)$, or equivalently, the observed landscape $U_{q}(x)$ ? This presents two possible challenges.

- The first is possibility that there are systems characterised by different $f(x)$ and different $g(x)$ that yield the same stationary probability distribution $P_{s}(x)$, and therefore, the same $U_{q}(x)$.

- A second challenge is that even if the deterministic system $f(x)$ is known, it may be possible that different choices of the noise component $g(x)$ could give rise to the same distribution $P_{s}(x)$. And similarly, there may be different deterministic systems $f(x)$ that can yield the same stationary distribution $P_{s}(x)$, by way of a fixed noise component $g(x)$.

It may not be surprising that a given stationary probability distribution can represent many different dynamical systems; indeed this has been previously shown empirically [19, 51. Nevertheless, it will be instructive to consider this more formally. Here we show that for onedimensional stochastic systems, the steady-state distribution $P_{s}(x)$ can always be obtained from systems characterised by different $f(x)$ and different $g(x)$, for any given stochastic integration convention $\alpha$. We demonstrate this result using an illustrative example, showing there are non-identifiability instances of the first kind (the first item above). We then discuss how non-identifiabilty can be resolved, thereby showing there do not exist nonidentifiability instances of the second kind (the second item above).

\section{Decomposition of the stationary probability distribution}

Fix some stationary probability distribution $P_{s}(x)$. Then for any noise component $g(x)$, and stochastic integration convention $\alpha$, the deterministic dynamics $f(x)$ can be solved as

$$
f(x)=\frac{1}{P_{s}(x)}\left[\frac{\partial}{\partial x}\left(\frac{g^{2}(x) P_{s}(x)}{2}\right)\right]-\alpha g(x) g^{\prime}(x) .
$$

On the other hand, given any deterministic dynamics $f(x)$, the noise component $g(x)$ can be solved as

$$
\begin{array}{r}
g(x)=\left[\frac{2}{1-\alpha}\left(P_{s}(x)\right)^{\frac{1}{(\alpha-1)}} \int^{x} f(z)\left(P_{s}(z)\right)^{\frac{-1}{(\alpha-1)}} d z\right]^{\frac{1}{2}} \\
\text { for } \alpha \in[0,1)
\end{array}
$$

and

$$
g(x)=\left[\frac{2}{P_{s}^{\prime}(x)} P_{s}(x) f^{\prime}(x)\right]^{\frac{1}{2}} \quad \text { for } \alpha=1 .
$$

Thus, the stationary probability distribution $P_{s}(x)$ can be obtained by any noise component $g(x)$ with the deterministic dynamics in Eq. (18), or by any deterministic component $f(x)$ and the multiplicative noise given in Eq. (19); the case where $\alpha=0$ (the Itô interpretation) can be found in Sura et al. 52 .

\section{Non-identifiability of the first kind}

In order to simplify our example, we will assume throughout that the stochastic integration convention $\alpha$ is known to be 0 , corresponding to the Itô interpretation. In practise, the stochastic interpretation $\alpha$ is often chosen a priori on the basis of the available information, but in general is not known and cannot be inferred from experimental data: there are different $f(x)$ and $\alpha$ that yield the same stationary probability distribution [10, 45, 49]. 
A.

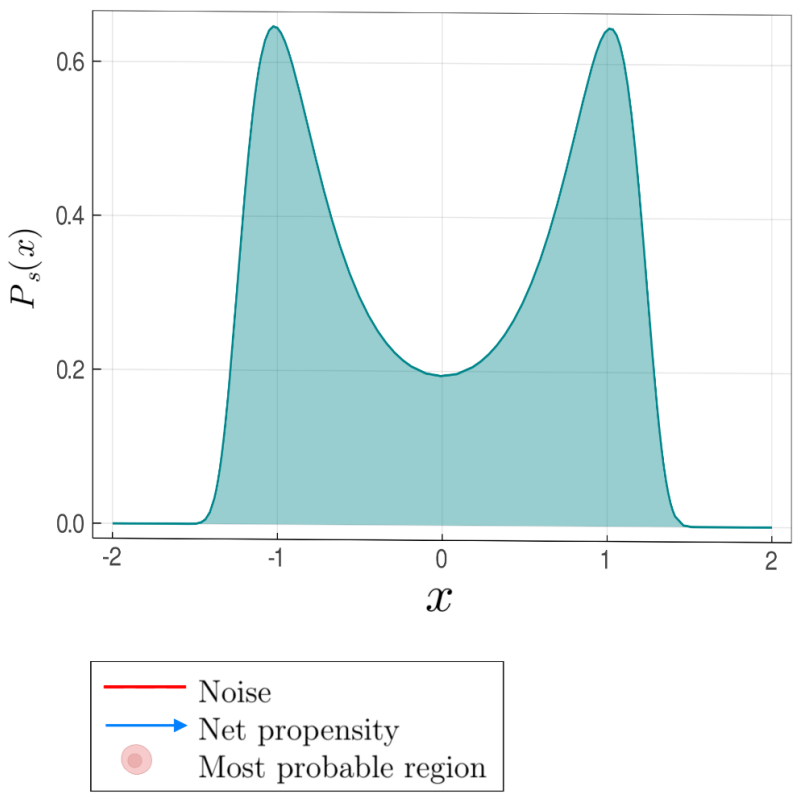

B.

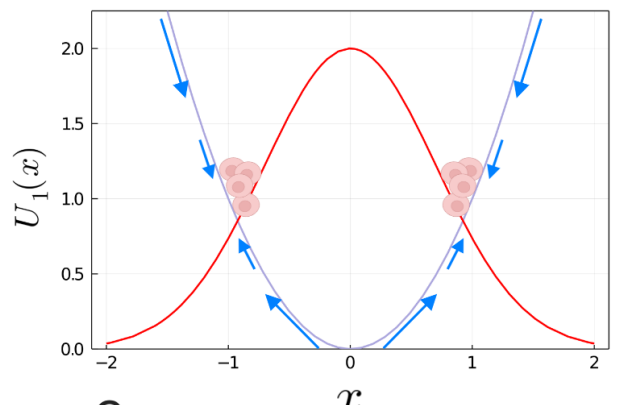

C.

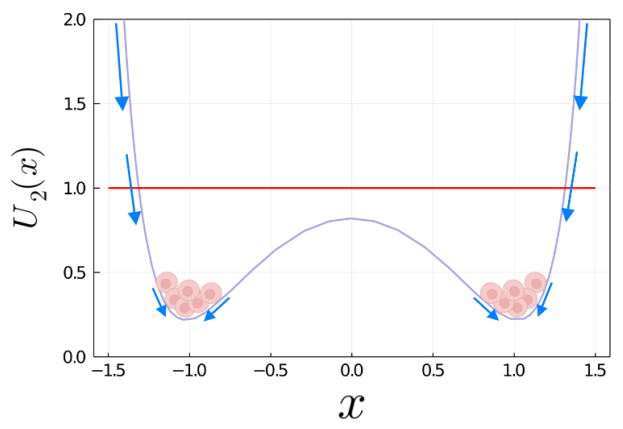

Figure 6: The same bimodal probability distribution (A) arises from a unimodal system subject to multiplicative noise (B), and from a bimodal system driven by additive noise $(\mathrm{C})$, resulting in non-identifiability of the first kind. In (B) the noise strength (red curve) is greater around the origin thereby pushing cells away from the origin and generating a bimodal probability distribution. (C) Additive noise does not shift the minima of $P_{s}(x)$ and so the distribution remains bimodal.

Consider the probability distribution obtained from the deterministic dynamics $f_{1}(x)=-x$ and the noise component $g_{1}(x)=\sigma \exp \left(-x^{2}\right)$

$$
P_{s}(x)=\frac{\mathcal{N}}{\sigma^{2}} \exp \left(2 x^{2}-\frac{1}{2 \sigma^{2}} \exp \left(2 x^{2}\right)\right)
$$

where $\mathcal{N}$ is the normalisation constant. This probability distribution is displayed in Fig. 6(A) for $\sigma=2$; note that the most probable states occur at $x_{\max }= \pm \sqrt{3 \log (2) / 2}$ $(\approx \pm 1.02)$. The corresponding potential function $U_{1}(x)=$ $x^{2}$ and noise function $g_{1}(x)$ are shown in Fig 6(B) as the purple and red curves, respectively. The net effect of noise on the potential function (as indicated by the blue arrows), as well as the most probable regions of the system's trajectory are also shown. As the noise is much higher at the stable stationary point relative to the strength of noise to the left and right of this point, cells are pushed away from the stable point, and the system's trajectory is more often found either side of the stable stationary point (the regions indicated by the pink cells). The result is the bimodal probability distribution given in Eq. 207.

Using the stationary probability decomposition above (Eq. 18), we are able to obtain a second system that gives rise to the same probability distribution given in Eq. 20. This system has substantially different dynamics: the underlying deterministic dynamics $f_{2}(x)=2 x-\frac{1}{4} x \exp \left(2 x^{2}\right)$ gives rise to the bistable system $U_{2}(x)$ shown in Fig. $6 \mathrm{C}$; note that the stable points of $U_{2}(x)$ occur at $x_{\max }=$ $\pm \sqrt{3 \log (2) / 2}$, which agree with the maxima of $P_{s}(x)$.
Moreover, the system is now perturbed only by the additive (constant) noise $g_{2}(x)=1$ (as opposed to the multiplicative noise $\left.g_{1}(x)\right)$. In contrast to Fig. 6B, we can see that the noise acts uniformly on the system's trajectory, and thus preserves the two stable points of the system. The result of the noise $g_{2}(x)$ on the potential $U_{2}(x)$ again produces the bimodal probability distribution given in Eq. 20.

Our example shows that having knowledge of the measured stationary distribution alone (or equivalently, the observed landscape) is not enough to infer the underlying regulatory dynamics of the stochastic system. Did the stationary distribution arise from a system with only one cell state perturbed by multiplicative noise, or a bistable system with only additive noise? In biological terms, knowing the typical distribution of cell states offers very little information about how a cell differentiates and travels (in terms of gene activity) to its cell fate.

\section{Non-identifiability of the second kind}

We now consider the second identifiability problem. To simplify our consideration, we again assume the stochastic integration convention $\alpha$ is known. Given the stationary probability distribution $P_{s}(x)$, and one of the components $f(x)$ or $g(x)$, we are then interested in whether we can uniquely identify the remaining component using only this information. The stationary probability decomposition above tells us (almost trivially) that knowledge 
of one of the components is sufficient to uniquely identify the other; uniqueness follows from the uniqueness property of first-order differential equations 53 (refer to the derivations of Eq.'s (18) and (19) in the supplementary material). We now briefly demonstrate this in the context of our previous example.

Assume again that we are given the stationary probability distribution in Fig. 6 (in practise, this distribution can be obtained from experimental data). Assume further that we now have some confidence in the underlying dynamics $f(x)$ of the system. For example, we have reason to believe the underlying system is bistable, and is described by $f(x)=2 x-\frac{1}{4} x \exp \left(2 x^{2}\right)$. Then from Eq. (19) the noise component $g(x)$ can be uniquely solved as

$$
\begin{aligned}
& g(x)=\left[\frac{8}{\mathcal{N}} \exp \left(2 x^{2}+\frac{1}{8} \exp \left(2 x^{2}\right)\right)\right. \\
& \left.\int^{x}\left(2 z-\frac{1}{4} z \exp \left(2 z^{2}\right)\right) \exp \left(2 x^{2}-\frac{1}{8} \exp \left(2 x^{2}\right)\right) d z\right]^{\frac{1}{2}}=1
\end{aligned}
$$

Thus, for one-dimensional stochastic systems, any one of $g(x), f(x)$ and $P_{s}(x)$ can be determined from knowledge of the other two. We remark that we have considered here only gradient systems (i.e. systems satisfying the relationship in Eq. (1)). If we broaden our scope to higher dimensions, which include non-gradient systems, then it has been shown (empirically) that there are non-identifiability instances of the second kind 19]: even when the noise component is fixed, there are systems defined by gradient and non-gradient deterministic dynamics that admit qualitatively indistinguishable stationary probability distributions.

\section{Stochastic bifurcations}

We have seen how noise may change the landscape qualitatively at critical parameter values: for example, transitioning from a bistable system to a monostable system. These changes can be viewed as binary branches on the Waddington landscape where cell-fate decisions take place, and may provide insight into how noise affects cell-fate decision making. At each branching point, a cell adopts one of two possible cell states [54, each defined by a unique gene expression profile or GRN. Mathematically, this point can be viewed as a bifurcation [2, 55, 56, 57. For deterministic dynamical systems a bifurcation results in a qualitative change of the solution (nature and number of fixed points) as a parameter, or set of parameters are varied 58 .

While bifurcation theory for deterministic dynamical systems is well-developed (and indeed extensively studied), the extension to its stochastic counterpart is perhaps less so. In stochastic dynamics there is potential for richer dynamics (see e.g. 59 and 60]), and we commonly distinguish between two types of bifurcations 61]: D-bifurcations, are the analogues of deterministic bifurcations; the 'D' stands for 'dynamical' and this type of bifurcation reflects sudden changes in the sign of the leading Lyapunov exponent of the dynamics [62. (P)bifurcations, by contrast, reflect sudden changes in the steady-state probability distribution over states. We have already seen examples of P-bifurcations: in Example 1 (resp. Fig. 6(B)) above, the probability density transitions from a bimodal (unimodal) to a unimodal (bimodal) distribution, by varying the noise intensity parameter. These changes, known as noise-induced transitions, indicate that the system around the P-bifurcation point is not structurally stable [61, 6], and show how the stochastic system can display qualitatively different behaviour from that of the deterministic system.

It is possible for $D$ - and $P$-bifurcations to occur individually or jointly, and the interplay between these two types of qualitative change in system behaviour is generally difficult to disentangle. Baxendale 63 for example, gives a dynamical system exhibiting a stochastic D-bifurcation, while the steady-state probability distribution is independent of the bifurcation parameter. On the other hand, Crauel and Flandoli 64] consider the stochastic system obtained from Eq. 8 in the presence of additive noise, and show that while the stationary probability density has a pitchfork bifurcation of equilibria, the dynamical structure of the system remains stable (i.e. the system does not exhibit a D-bifurcation). As static measures are unable to capture the full dynamics of a system, the P-bifurcation has been deemed too limiting an approach to fully explore the behaviour of a stochastic dynamical system [61, 64]; refer to Box 3 for a list of shortcomings. The dynamical approach may therefore offer a richer understanding of the dynamical structure of a system than the phenomenological approach alone. Indeed, a number of biological and gene regulatory studies have emerged that investigate the dynamical behaviour of a stochastic system from both perspectives 65, 66. For a comprehensive analysis on $\mathrm{P}$ - and D-bifurcations in one-dimensional dynamical systems, we refer the reader to [61, and for an explicit consideration of noise-induced transitions (restricted to P-bifurcations only) we recommend the extensive work of 11 .

\section{Discussion}

Here we provide an analytically rigorous, yet intuitive and instructive demonstration of the subtleties that may arise, and the consequences thereof, when computing the landscape in the presence of noise. While some of these complexities may be well-appreciated in certain fields, considering the diverse audience the probabilistic landscape may appeal to, it is important to offer this mathematical framework in a more digestible manner.

We consider how different noise types affect the landscape. In particular, we demonstrate the oft-observed finding that additive noise results only in a disorganising effect on the landscape. In the presence of multiplicative noise, the effects may be more profound, resulting in significant deviations between the deterministic and proba- 
bioRxiv preprint doi: https://doi.org/10.1101/2020.12.21.423724; this version posted December 22, 2020. The copyright holder for this

\section{Box 3 | Stochastic Bifurcation Theory}

Bifurcations: deterministic to stochastic

A bifurcation is defined as a qualitative change in the behaviour of a dynamical system in response to varying a parameter, or set of parameters; the bifurcation parameter(s) [58. Bifurcations are related to the arrival of new solutions and their relative stability. While bifurcation theory has been extensively developed for deterministic systems, relatively little progress has been made towards extending the theory to stochastic dynamical systems.

\section{Approaching stochastic bifurcations}

Much of the work so far in developing a bifurcation theory for stochastic dynamical systems has been made by Arnold 61, where stochastic bifurcations are distinguished as either "static" (based on the FPE) or "dynamic" (based on the Lyponent exponents). More specifically, stochastic bifurcations may be viewed in terms of: (i) Phenomonological (P)-bifurcations that study qualitative changes in the steady-state probability distribution of a stochastic dynamical system (e.g. from unimodal to bimodal); and (ii) Dynamical (D)-bifurcations that study the stability of the dynamical structure of a stochastic system with respect to so-called invariant measures - stochastic analogues of fixed points. The stochastic D-bifurcation is identified as a sign change in the maximal Lyapunov exponent. The two notions are independent of one another and do not necessairly occur together [63, 64]

\section{P- or D- bifurcation?}

In the same way that studying the steady-state probability distribution has limitations in inferring the dynamics of a given system, P-bifurcations too limit our understanding of stochastic dynamical systems. Specifically, they present the following significant disadvantages [61]:

- Restricted to the study of white (i.e. Markovian) noise;

- Unrelated to the stability of the dynamical system i.e. qualitative changes in the steady-state probability distribution generally don't reflect changes in the system's stability;

- Potentially misses bifurcations represented by non-Markovian invariant measures.

Subsequently, D-bifurcations may offer a richer understanding of the dynamics in comparison to P-bifurcations. Thus, in order to obtain a better understanding of the biological behaviour of cell-fate decisions, it may prove beneficial to consider both types of stochastic bifurcations in the analyses of a stochastic dynamical system.

bilistic landscapes. While these deviations are to some extent noted in the landscape literature [47, 23], we believe that the reason for them is less clear, often buried in a mound of mathematical jargon, rendering the rationale inaccessible to many of us who need it. In short, there is a subtle interplay between the stochastic and deterministic components, with the resultant probabilistic landscape arising as the balance of these influences. It is perhaps unsurprising that significant distortion of the topological features of the landscape can arise when these influences are in conflict. By varying the stochastic component on a fixed deterministic system, we show that the fixed points of the system may be preserved, shifted or destroyed. Sometimes even new fixed points can emerge. It is exactly these changes that are associated with stochastic P-bifurcations. We point out that P-bifurcations may be limited in capturing the full dynamics of the underlying stochastic system and we may therefore additionally want to consider D-bifurcations - the natural analogue of deterministic bifurcations.
A further subtlety arises from the nuanced mathematical treatment of stochasticity in the form of white noise, specifically when it enters the system multiplicatively. We show that the two most commonly employed stochastic integration conventions can produce qualitatively different steady-state distributions and subsequently, different probabilistic landscapes. As these deviations are expected to be significant in modelling real-world biological systems, we hope that this emphasises the importance in considering a stochastic differential equation in conjunction with a stochastic calculus when constructing the probabilistic landscape.

While additive noise is often employed in practise [19, 23, 67] - perhaps because it mitigates the intricacies of stochastic calculus and the associated consequences discussed herein - it does not satisfy two of the most commonly observed features of noise in cellular differentiation: (i) that the noise is high near the bifurcation point or unstable equilibria, and (ii) is comparatively lower at the differentiated cell states or stable equilibria [41. A natu- 
bioRxiv preprint doi: https://doi.org/10.1101/2020.12.21.423724; this version posted December 22, 2020. The copyright holder for this preprint (which was not certified by peer review) is the author/funder, who has granted bioRxiv a license to display the preprint in perpetuity. It is made available under aCC-BY 4.0 International license.

ral, and more sophisticated noise form that satisfies these properties is when the noise is proportional to the deterministic landscape itself, or more generally, if it observes a power relationship to the deterministic landscape. We show that in these instances, the fixed points of the underlying deterministic system will be preserved by the probabilistic landscape. Thus, in situations where it is desirable to preserve the dynamical information of the deterministic dynamics, it may not be necessary to turn to alternate methods such as those presented in [47] to compute the landscape, which may be complicated to implement in practice.

An important consideration is the identifiability of the deterministic dynamics in the presence of noise. We show that one-dimensional stochastic systems can be characterised by different deterministic and different stochastic components that give rise to the same stationary distribution. Thus, even if the probabilistic landscape is obtained, we cannot make definitive assumptions about the underlying deterministic and stochastic components of the system on the basis of this information alone. Despite this, non-identifiability may be resolved (at least for onedimensional systems) by obtaining additional information on any one of the other components of the system, provided we fix a priori a stochastic integration convention.

While the examples discussed herein are for onedimensional gradient systems, we might not be completely discouraged by this since gradient systems are representative of more general dynamical systems. Furthermore, there may be cases were a significant reduction in the dimensionality of the data may preserve the basic dynamics of the system, allowing us to still gain meaningful insight into the high-dimensional dynamics [55, 68, 69]. We hope that this careful and explicit consideration of noise on the potential landscape will draw the researcher's attention to the associated complexities and limitations. These are not merely fringe technicalities that can be ignored, but rather significant mathematical caveats with fundamental implications for constructing the landscape as the evolution of a dynamical system in the presence of stochastic fluctuations.

\section{Competing interests}

The authors declare they have no competing interests.

\section{Acknowledgments}

We gratefully acknowledge Rowan D. Brackston for helpful discussions in the early stages of this research, as well the support from the members of the Theoretical Systems Biology Group at the University of Melbourne. L.H. and M.P.H.S. are supported by the University of Melbourne DVCR fund. M.A.C is supported by the University of Melbourne graduate research scholarship.

\section{References}

[1] J. E. Ferrell Jr, Bistability, Bifurcations, and Waddington's Epigenetic Landscape, Current Biology 22 (2012) R458R466. URL: https://linkinghub.elsevier.com/retrieve/ pii/S0960982212003326 doi 10.1016/j.cub.2012.03.045

[2] N. Moris, C. Pina, A. M. Arias, Transition states and cell fate decisions in epigenetic landscapes, Nat. Rev. Genet. 17 (2016) 693-703.

[3] C. H. Waddington, The strategy of the genes, Allen \& Unwin, London, 1957.

[4] R. Thom, Structural stability and morphogenesis: an outline of a general theory of models, Addison-Wesley Pub., Reading, Mass., 1989.

[5] K. Erguler, M. P. H. Stumpf, Statistical interpretation of the interplay between noise and chaos in the stochastic logistic map., Mathematical biosciences 216 (2008) 90-99. doi 10.1016/ j.mbs.2008.08.012

[6] M. Demazure, Bifurcations and catastrophes: geometry of solutions to nonlinear problems, Universitext, Springer, Berlin, 2000. URL: http://www.loc.gov/catdir/enhancements/ fy0815/99053117-d.html

[7] J. Jost, Riemannian geometry and geometric analysis, Universitext, 6th ed., Springer, Heidelberg, 2011.

[8] S. Smale, Differential dynamical systems, Bull Am Math Soc 73 (1967) 747-817.

[9] R. Bott, Morse theory indomitable, Publications mathématiques de II.H.É.S. 68 (1988) 99-114. URL: http: //www.numdam.org/article/PMIHES_1988__68_-99_0.pdf

[10] C. Gardiner, Stochastic Methods: A Handbook For The Natural And Social Sciences, Springer, 2009.

[11] W. Horsthemke, R. Lefever, Noise-Induced Transitions: Theory and Applications in Physics, Chemistry, and Biology, Springer Series in Synergetics, Springer Berlin Heidelberg, 2006.

[12] J. X. Zhou, M. D. S. Aliyu, E. Aurell, S. Huang, Quasi-potential landscape in complex multi-stable systems, Journal of the Royal Society Interface 9 (2012) 35393553. URL: http://rsif.royalsocietypublishing.org/cgi/ doi/10.1098/rsif.2012.0434 doi 10.1098/rsif.2012.0434

[13] P. Zhou, T. Li, Construction of the landscape for multi-stable systems: Potential landscape, quasi-potential, A-type integral and beyond, The Journal of Chemical Physics 144 (2016) 094109. URL: https://doi.org/10.1063/1.4943096 doi 10 . 1063/1.4943096

[14] A. Guillemin, M. P. H. Stumpf, Non-equilibrium statistical physics, transitory epigenetic landscapes, and cell fate decision dynamics, Mathematical Biosciences and Engineering 17 (2020) 7916-7930. URL: http://arxiv.org/abs/2011.04252v1.

[15] J. Wang, L. Xu, E. Wang, Potential landscape and flux framework of nonequilibrium networks: Robustness, dissipation, and coherence of biochemical oscillations, PNAS 105 (2008) 12271-12276. URL: https://www.pnas.org/ content/105/34/12271 doi $10.1073 /$ pnas.0800579105 arXiv: https://www.pnas.org/content/105/34/12271.full.pdf

[16] J. Wang, L. Xu, E. Wang, S. Huang, The potential landscape of genetic circuits imposes the arrow of time in stem cell differentiation, Biophys. J. 99 (2010) 29-39.

[17] S. Lapidus, B. Han, J. Wang, Intrinsic noise, dissipation cost, and robustness of cellular networks: The underlying energy landscape of mapk signal transduction, PNAS 105 (2008) 6039-6044. URL: https://ww.pnas. org/content/105/16/6039 doi $10.1073 /$ pnas.0708708105 arXiv:https://www.pnas.org/content/105/16/6039.full.pdf

[18] Z. XM, Y. L, H. L, A. P., Robustness, stability and efficiency of phage lambda genetic switch: dynamical structure analysis, J. Bioinform. Comput. Biol. 2 (2004) 785-817. doi $10.1142 / \mathrm{s} 0219720004000946$

[19] R. D. Brackston, E. Lakatos, M. P. H. Stumpf, Transition state characteristics during cell differentiation., PLoS computat, biol. 14 (2018) e1006405. doi 10.1371/journal.pcbi.1006405.

[20] C. Li, T. Hong, Q. Nie, Quantifying the landscape and kinetic 
bioRxiv preprint doi: https://doi.org/10.1101/2020.12.21.423724; this version posted December 22, 2020. The copyright holder for this preprint (which was not certified by peer review) is the author/funder, who has granted bioRxiv a license to display the preprint in perpetuity. It is made available under aCC-BY 4.0 International license.

paths for epithelial-mesenchymal transition from a core circuit, Phys. Chem. Chem. Phys. 18 (2016) 17949-17956.

[21] C. Lv, X. Li, F. Li, T. Li, Constructing the energy landscape for genetic switching system driven by intrinsic noise, PLOS ONE 9 (2014) 1-10. URL: https://doi.org/10.1371/journal.pone. 0088167 doi $10.1371 /$ journal.pone.0088167

[22] C. Li, E. Wang, J. Wang, Potential landscape and probabilistic flux of a predator prey network, PLOS ONE 6 (2011) 1-9. URL: https://doi.org/10.1371/journal.pone.0017888 doi 10.1371/journal.pone.0017888

[23] R. D. Brackston, A. Wynn, M. P. H. Stumpf, Construction of quasipotentials for stochastic dynamical systems: An optimization approach, Phys. Rev. E 98 (2018) 022136. doi 10.1103/ PhysRevE.98.022136

[24] A. Richard, L. Boullu, U. Herbach, A. Bonnafoux, V. Morin, E. Vallin, A. Guillemin, N. Papili Gao, R. Gunawan, J. Cosette, O. Arnaud, J.-J. Kupiec, T. Espinasse, S. Gonin-Giraud, O. Gandrillon, Single-Cell-Based analysis highlights a surge in Cell-to-Cell molecular variability preceding irreversible commitment in a differentiation process, PLoS Biol. 14 (2016) e1002585.

[25] M. Mojtahedi, A. Skupin, J. Zhou, I. G. Castaño, R. Y. Y. Leong-Quong, H. Chang, K. Trachana, A. Giuliani, S. Huang, Cell fate decision as high-dimensional critical state transition, PLoS Biol. 14 (2016) e2000640.

[26] P. S. Stumpf, R. C. G. Smith, M. Lenz, A. Schuppert, F.-J Müller, A. Babtie, T. E. Chan, M. P. H. Stumpf, C. P. Please, S. D. Howison, F. Arai, B. D. MacArthur, Stem cell differentiation as a non-markov stochastic process, Cell. Syst. 5 (2017) 268-282.e7.

[27] S. Semrau, J. E. Goldmann, M. Soumillon, T. S. Mikkelsen, R. Jaenisch, A. van Oudenaarden, Dynamics of lineage commitment revealed by single-cell transcriptomics of differentiating embryonic stem cells, Nat. Commun. 8 (2017) 1096.

28] A. Moussy, J. Cosette, R. Parmentier, C. da Silva, G. Corre, A. Richard, O. Gandrillon, D. Stockholm, A. Páldi, Integrated time-lapse and single-cell transcription studies highlight the variable and dynamic nature of human hematopoietic cell fate commitment, PLoS Biol. 15 (2017) e2001867.

[29] M. B. Elowitz, A. J. Levine, E. D. Siggia, P. S. Swain, Stochastic gene expression in a single cell, Science 297 (2002) 1183-1186.

30] M. Kærn, T. C. Elston, W. J. Blake, J. J. Collins, Stochasticity in gene expression: from theories to phenotypes, Nature Reviews Genetics 6 (2005) 451-464. URL: https://doi.org/10. $1038 / \mathrm{nrg} 1615$ doi $10.1038 / \mathrm{nrg} 1615$

[31] M. H. H. Arkin A, Ross J, Stochastic kinetic analysis of developmental pathway bifurcation in phage lambda-infected escherichia coli cells, Genetics 149 (1998) 1633-48.

[32] T. Kalmar, C. Lim, P. Hayward, S. Muñoz-Descalzo, J. Nichols, J. Garcia-Ojalvo, A. Martinez Arias, Regulated fluctuations in nanog expression mediate cell fate decisions in embryonic stem cells, PLOS Biology 7 (2009) 1-16. URL: https://doi.org/10.1371/journal.pbio.1000149 doi $10.1371 /$ journal.pbio.1000149

[33] A. Guillemin, M. P. Stumpf, Noise and the molecular processes underlying cell fate decision-making, Physical Biology (2020). URL: http://iopscience.iop.org/article/10.1088/ 1478-3975/abc9d1

[34] P. S. Swain, M. B. Elowitz, E. D. Siggia, Intrinsic and extrinsic contributions to stochasticity in gene expression, Proc. Natl. Acad. Sci. 99 (2002) 12795-12800. doi 10.1073/pnas. 162041399

[35] M. Assaf, E. Roberts, Z. Luthey-Schulten, N. Goldenfeld, Extrinsic noise driven phenotype switching in a self-regulating gene, Phys. Rev Lett. (2013).

[36] L. Ham, D. Schnoerr, R. D. Brackston, M. P. H. Stumpf, Exactly solvable models of stochastic gene expression, J. Chem. Phys. 152 (2020) 144106.

[37] L. Ham, R. D. Brackston, M. P. H. Stumpf, Extrinsic Noise and Heavy-Tailed Laws in Gene Expression., Phys. Rev. Lett. 124 (2020) 108101. doi 10.1103/PhysRevLett.124.108101
38] S. Okawa, S. Nicklas, S. Zickenrott, J. C. Schwamborn, A. Del Sol, A generalized gene-regulatory network model of stem cell differentiation for predicting lineage specifiers., Stem Cell Rep. 7 (2016) 307-315. URL: https:// linkinghub.elsevier.com/retrieve/pii/S2213671116301382 doi $10.1016 /$ j.stemcr.2016.07.014

[39] T. E. Chan, M. P. H. Stumpf, A. C. Babtie, Gene Regulatory Network Inference from Single-Cell Data Using Multivariate Information Measures., Cell Sys. 5 (2017) 251-267.e3.

[40] A. B. Bloom, M. H. Zaman, Influence of the microenvironment on cell fate determination and migration, Physiol. Genomics 46 (2014) 309-314.

[41] E. Pujadas, A. P. Feinberg, Regulated noise in the epigenetic landscape of development and disease, Cell 148 (2012) 11231131.

[42] A. Hilfinger, J. Paulsson, Separating intrinsic from extrinsic fluctuations in dynamic biological systems, Proc. Natl. Acad. Sci. U. S. A. 108 (2011) 12167-12172.

[43] N. P. Gao, O. Gandrillon, A. Paldi, U. Herbach, R. Gunawan, Universality of cell differentiation trajectories revealed by a reconstruction of transcriptional uncertainty landscapes from single-cell transcriptomic data, bioRxiv (2020).

[44] G. Volpe, J. Wehr, Effective drifts in dynamical systems with multiplicative noise: a review of recent progress, Rep. Prog. Phys. 79 (2016) 053901.

[45] R. Mannella, P. V. E. McClintock, Itô versus stratonovich: 30 years later, Fluct. Noise Lett. 11 (2012) 1240010

[46] G. Pesce, A. McDaniel, S. Hottovy, J. Wehr, G. Volpe, Stratonovich-to-itôtransition in noisy systems with multiplicative feedback, Nat. Commun. 4 (2013) 2733. URL: https: //doi.org/10.1038/ncomms3733 doi 10.1038/ncomms3733

[47] Y. Tang, R. Yuan, G. Wang, X. Zhu, P. Ao, Potential landscape of high dimensional nonlinear stochastic dynamics with large noise, Sci. Rep. 7 (2017) 15762.

[48] J. Gunawardena, Models in biology: 'accurate descriptions of our pathetic thinking',, BMC biology 12 (2014) 29. URL: http://www.biomedcentral.com/1741-7007/12/29. doi $10.1186 / 1741-7007-12-29$

[49] N. G. van Kampen, Itô versus stratonovich, J. Stat. Phys. 24 (1981) $175-187$

[50] A. L. MacLean, T. Hong, Q. Nie, Exploring intermediate cell states through the lens of single cells, Current Opinion in Systems Biology 9 (2018) 32 - 41. URL: http://www.sciencedirect.com/science/article/pii/ S2452310017302238 doi/https://doi.org/10.1016/j.coisb. 2018.02.009 mathematic modelling.

[51] C. Weinreb, S. Wolock, B. K. Tusi, M. Socolovsky, A. M. Klein, Fundamental limits on dynamic inference from single-cell snapshots, PNAS 115 (2018) E2467-E2476. URL: https://www.pnas.org/ content/115/10/E2467 doi $10.1073 /$ pnas.1714723115. arXiv:https://www.pnas.org/content/115/10/E2467.full.pdf

[52] P. Sura, M. Newman, C. Penland, P. Sardeshmukh, Multiplicative noise and Non-Gaussianity: A paradigm for atmospheric regimes?, Journal of Atmospheric Sciences 62 (2005) 1391-1409.

[53] V. I. Arnold, Ordinary differential equations, The MIT Press, 1998.

[54] P. Hayward, T. Kalmar, A. M. Arias, Wnt/Notch signalling and information processing during development, Development 135 (2008) 411-424.

[55] E. Marco, R. L. Karp, G. Guo, P. Robson, A. H. Hart, L. Trippa, G.-C. Yuan, Bifurcation analysis of single-cell gene expression data reveals epigenetic landscape, Proc. Natl. Acad. Sci. U. S. A. 111 (2014) E5643-50.

[56] J. Chen, A. Schlitzer, S. Chakarov, F. Ginhoux, M. Poidinger, Mpath maps multi-branching single-cell trajectories revealing progenitor cell progression during development, Nat. Commun. 7 (2016) 11988.

[57] C. Trapnell, D. Cacchiarelli, J. Grimsby, P. Pokharel, S. Li, M. Morse, N. J. Lennon, K. J. Livak, T. S. Mikkelsen, J. L. Rinn, The dynamics and regulators of cell fate decisions are revealed 
[58] J. Jost, Dynamical Systems Examples Of Complex Behaviour, Springer, 2005.

[59] D. Zhao, S. Yuan, Noise-induced bifurcations in the stochastic chemostat model with general nutrient uptake functions, Applied Mathematics Letters 103 (2020). URL: https://www.sciencedirect.com/science/article/pii/ S0893965919305063

60] M. Mendler, J. Falk, B. Drossel, Analysis of stochastic bifurcations with phase portraits, PloS one 13 (2018) e0196126. URL: https://dx.plos.org/10.1371/journal.pone.0196126 doi $10.1371 /$ journal.pone. 0196126

[61] L. Arnold, Random Dynamical Systems, 1st ed., Springer, Springer-Verlag Berlin Heidelberg, 1998.

[62] P. Glendinning, Stability, instability, and chaos: an introduction to the theory of nonlinear differential equations, Cambridge University Press, Cambridge, 1994.

[63] P. H. Baxendale, Asymptotic behaviour of stochastic flows of diffeomorphisms, in: Stochastic Processes and Their Applications, Springer Berlin Heidelberg, 1986, pp. 1-19.

[64] H. Crauel, F. Flandoli, Additive noise destroys a pitchfork bifurcation, J. Dynam. Differential Equations 10 (1998) 259-274.

[65] A. Zakharova, J. Kurths, T. Vadivasova, A. Koseska, Analysing dynamical behavior of cellular networks via stochastic bifurcations, PLoS One 6 (2011) e19696.

[66] Z. Huang, Q. Yang, J. Cao, Stochastic stability and bifurcation for the chronic state in marchuk's model with noise, Applied Mathematical Modelling 35 (2011) 5842 - 5855. URL: http://www.sciencedirect.com/ science/article/pii/S0307904X11003350 doi/https: //doi.org/10.1016/j.apm.2011.05.027.

[67] X. Qiu, S. Ding, T. Shi, From understanding the development landscape of the canonical fate-switch pair to constructing a dynamic landscape for two-step neural differentiation, PLOS ONE 7 (2012) e49271.

[68] C. Li, J. Wang, Quantifying cell fate decisions for differentiation and reprogramming of a human stem cell network: landscape and biological paths, PLoS Comput. Biol. 9 (2013) e1003165.

[69] X. Zhang, K. H. Chong, L. Zhu, J. Zheng, A monte carlo method for in silico modeling and visualization of waddington's epigenetic landscape with intermediate details, Biosystems 198 (2020) 104275. URL: http://www.sciencedirect. com/science/article/pii/S030326472030157X doilhttps : //doi.org/10.1016/j.biosystems.2020.104275 


\title{
Supplementary material for:
}

\section{Shaping the Epigenetic Landscape: Complexities and Consequences}

\author{
Abstract \\ Here we provide details and proofs of the results given in Shaping the Epigenetic Landscape: Complexities \\ and Consequences.
}

\section{Derivation of the general steady-state probability distribution}

We begin by providing full details of the derivation to Eq. (6) of the main text. The solution is well known, but we include the derivation for the sake of completeness. Consider first the Langevin Equation of a one-dimensional stochastic differential equation (SDE), where the dependence on the stochastic integration convention $\alpha$ is explicit,

$$
d x=\left(f(x)+\alpha g(x) \frac{\partial g(x)}{\partial x}\right) d t+g(x) d W_{t} .
$$

The associated Fokker-Planck equation (FPE) [1] is obtained by inspection of (1), and can be written as

$$
\frac{\partial P(x, t)}{\partial t}=-\frac{\partial}{\partial x}\left[\left(f(x)+\alpha g(x) \frac{\partial g(x)}{\partial x}\right) P(x, t)\right]+\frac{\partial^{2}}{\partial x^{2}}\left[\frac{g^{2}(x)}{2} P(x, t)\right] .
$$

The steady-state solution, denoted $P_{s}(x)$, is obtained from Eq. (2) by setting $\frac{\partial P(x, t)}{\partial x}=0$. The equation for $P_{s}(x)$ may then be written as

$$
\frac{d}{d x}\left[-\left(f(x)+\alpha g(x) g^{\prime}(x)\right) P_{s}(x)+\frac{d}{d x}\left[\frac{g^{2}(x)}{2} P_{s}(x)\right]\right] \equiv \frac{d}{d x}(J(x))=0,
$$

where $g^{\prime}(x):=\frac{d}{d x}(g(x))$. Here $J$ denotes the probability flux, and is easily seen from (3) to be constant. If $J$ is 0 everywhere (i.e. the system satisfies the detailed balance condition [1]), then Eq. (3) reduces to

$$
-\left(f(x)+\alpha g(x) g^{\prime}(x)\right) P_{s}(x)+\frac{d}{d x}\left[\frac{g^{2}(x)}{2} P_{s}(x)\right]=0 .
$$

We now make the substitution

$$
Y(x)=g^{2}(x) P_{s}(x) .
$$

Substituting Eq. (5) into Eq. (4) we obtain the following equation in $Y$,

$$
-\left(f(x)+\alpha g(x) g^{\prime}(x)\right) \frac{Y(x)}{g^{2}(x)}+\frac{d}{d x}\left[\frac{Y(x)}{2}\right]=0,
$$

which is simply a first-order linear homogeneous differential equation, and is easily solved as

$$
Y(x)=C \exp \left(2 \int^{x} \frac{f(z)+\alpha g(z) g^{\prime}(z)}{g^{2}(z)} d z\right)
$$

where $C \in \mathbb{R}$. It follows that the general form of the steady-state solution of the FPE is given by:

$$
P_{s}(x)=\frac{\mathcal{N}}{g^{2}(x)} \exp \left(2 \int^{x} \frac{f(z)+\alpha g(z) g^{\prime}(z)}{g^{2}(z)} d z\right),
$$




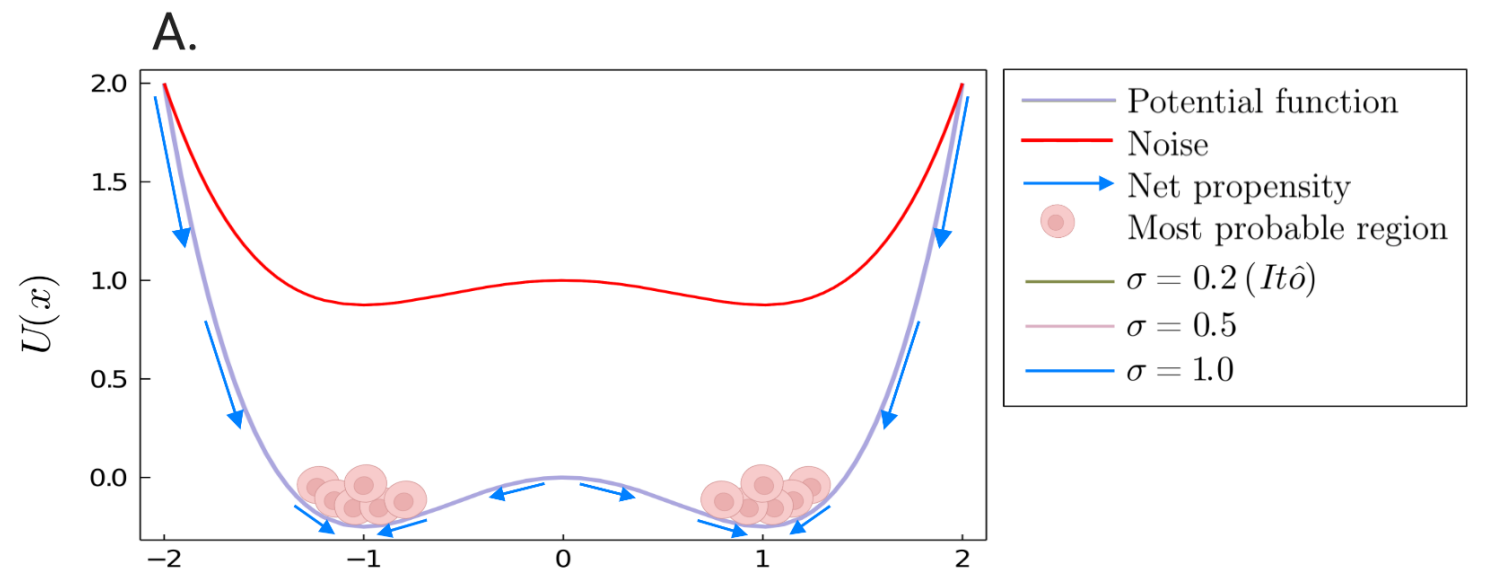

B.

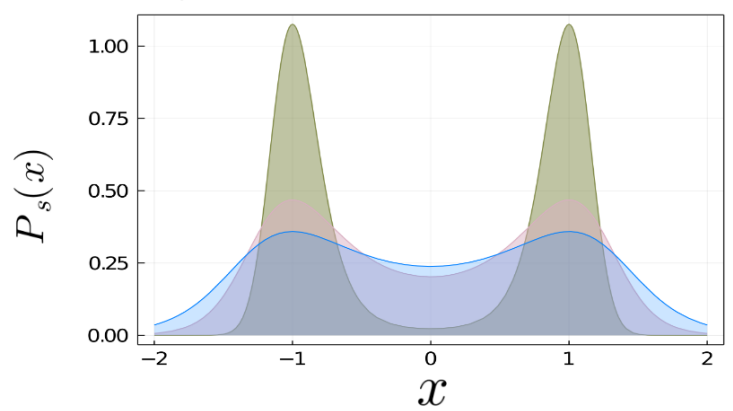

C.

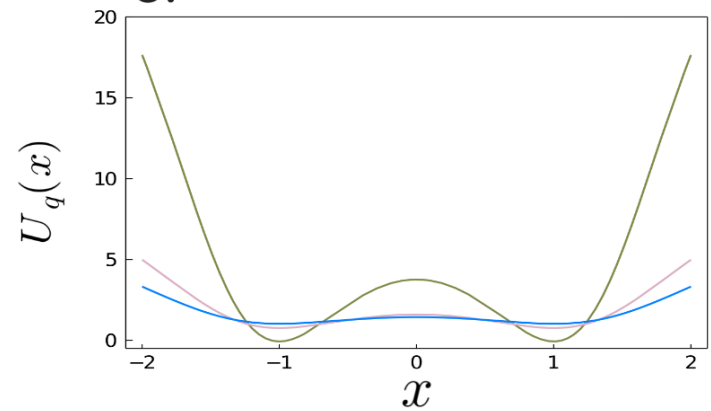

Figure 1: (A) A schematic of the system's trajectory. Here we consider a deterministic double-well potential $U(x)$ driven by multiplicative noise satisfying the power relationship given in Eq. 8) with $C=2$ and $\delta=1$. (B\&C) Analytical steady-state probability distribution $P_{s}(x)$ for the Ito interpretation $\alpha=0$. We consider the effects of increasing strengths of multiplicative noise $g(x)=\sigma(U(x)+2)$, where $\sigma=0.2$ (green curve), 0.5 (pink curve), 1.0 (blue curve). As $\sigma$ increases, the maxima of $P_{s}(x)$ and minima of $U_{q}(x)$ remain fixed.

where $\mathcal{N}$ is the normalisation constant. This is Eq. (6) in the main text. It then follows immediately, from Eq. (3) in the main text, that the probabilistic landscape $U_{q}(x)$ (Eq. (7) in the main text), is given by

$$
U_{q}(x)=\ln \left(\frac{g^{2}(x)}{\mathcal{N}}\right)-2 \int^{x} \frac{f(z)+\alpha g(z) g^{\prime}(z)}{g^{2}(z)} d z .
$$

\section{Multiplicative noise}

\section{Preserving fixed points under multiplicative noise}

In the main text, we claim that if the noise component $g(x)$ is related to the potential landscape $U(x)$ by way of the following power relationship,

$$
g(x)=\sigma(U(x)+C)^{\delta},
$$

where $\sigma>0$ is the noise intensity and $C, \delta$ are any non-negative real numbers, then provided that $U(x)+C$ is strictly positive, the fixed points of $U_{q}(x)$ will correspond precisely to those of $U(x)$, when they exist. Here we provide a proof of this result. First we remind the reader that since we are working with gradient systems, the deterministic component $f(x)$ is equal to the negative gradient of the potential $U(x)$ (see Eq. (1) of the main text). The proof proceeds in two cases: $(1) \alpha \in[0,1)$ and (2) $\alpha=1$. We begin by assuming that 
$\alpha \in[0,1)$, then from the general solution for $P_{s}(x)($ Eq. $(6)$ ) we have,

$$
P_{s}(x)=\frac{\mathcal{N}}{\sigma^{2}(U(x)+C)^{2 \delta}} \exp \left(\frac{2}{\sigma^{2}} \int^{x} \frac{\left(\alpha \delta \sigma^{2}(U(z)+C)^{2 \delta-1}-1\right) U^{\prime}(z)}{(U(z)+C)^{2 \delta}} d z\right) .
$$

Now letting $v=U(x)+C$ so that $v^{\prime}=U^{\prime}(x)$, the integral in Eq. (9) becomes

$$
\frac{2}{\sigma^{2}} \int^{U(x)+C} \frac{\alpha \delta \sigma^{2}}{v}-\frac{1}{v^{2 \delta}} d v=\frac{2}{\sigma^{2}}\left(\alpha \delta \sigma^{2} \ln (U(x)+C)-\frac{1}{(-2 \delta+1)}(U(x)+C)^{-2 \delta+1}+D\right),
$$

for $\delta \neq \frac{1}{2}$, and

$$
8 \int^{U(x)+C}\left(\frac{\alpha \sigma^{2}}{2}-1\right) \frac{1}{v} d v=8\left(\frac{\alpha \sigma^{2}}{2}-1\right)(\ln (U(x)+C)+D)
$$

for $\delta=\frac{1}{2}$, where $D$ is some constant.

When $\delta=\frac{1}{2}$, calculating $P_{s}^{\prime}(x)$ it is easy to show that $P_{s}^{\prime}(x)=0$ is equivalent to $U^{\prime}(x)=0$. We now consider the case where $\delta \neq \frac{1}{2}$. Then Eq. 90 becomes,

$$
P_{s}(x)=\frac{\mathcal{N}}{\sigma^{2}} \exp \left(2 \delta(\alpha-1) \ln (U(x)+C)-\frac{2}{\sigma^{2}(-2 \delta+1)}(U(x)+C)^{-2 \delta+1}+\frac{2 D}{\sigma^{2}}\right)
$$

Then calculating $P_{s}^{\prime}(x)$, we have that $P_{s}^{\prime}(x)=0$ is equivalent to

$$
U^{\prime}(x)\left(\sigma^{2} \delta(1-\alpha)(U(x)+C)^{2 \delta-1}+1\right)=0
$$

If $\delta=0$, this reduces to $U^{\prime}(x)=0$, and we are done. Now, assume that $\delta>0$. It then follows from Eq. 111) that either $U^{\prime}(x)=0$ or

$$
(U(x)+C)^{2 \delta-1}=-\frac{1}{\sigma^{2} \delta(1-\alpha)} .
$$

As $\alpha \in[0,1)$ we have that $(1-\alpha) \in(0,1]$, and since $\delta>0$, the right hand side of Eq. (12) is negative. Now, as $U(x)+C$ is strictly positive by assumption, it follows that Eq. 12 has no solutions. Thus, the stationary points of $P_{s}(x)$, and therefore of $U_{q}(x)$, will agree with those of $U(x)$ when they exist. The case for $\alpha=1$ is similar. The proof proceeds in same way as for Case (1), except that now Eq. (11) reduces to $U^{\prime}(x)=0$, and so we immediately obtain the desired result.

We now briefly consider an example of noise satisfying the power relationship in Eq. (8) acting on the double-well potential $U(x)$ (defined in Eq. (8) of the main text). It can be shown that the steady-state probability distribution for the state of the system $x$ is given by

$$
P_{s}(x)=\frac{4 \mathcal{N}}{\sigma^{2}\left(x^{4}-2 x^{2}+8\right)^{2}} \exp \left(\frac{8}{\sigma^{2}}\left(\frac{1}{x^{4}-2 x^{2}+8}+C\right)\right) .
$$

In Fig. 1(A), we display the effects of the noise $g(x)=\sigma(U(x)+2)$ on the double-well potential $U(x)$. In Fig. 1 $(B \& C)$, we display the steady-state distribution and corresponding landscape for the Itô interpretation (the Stratonovich interpretation produces very similar results). We can see that the effect of noise is qualitatively indistinguishable from that of additive noise (compare with Fig. 2 of the main text).

\section{Example 1: Shifting fixed points}

In the main text, the first example of multiplicative noise is for the noise component $g(x)=\frac{\sigma}{4}\left(x^{4}-x^{2}+8\right)$. Here we provide details of the derivation of the stationary probability distribution, and corresponding probabilistic landscape. From the general solution to the steady-state probability distribution $P_{s}(x)$ (Eq. (6) in the main text), with $f(x)=x-x^{3}$ and $g(x)=\frac{\sigma}{4}\left(x^{4}-x^{2}+8\right)$, we have

$$
\begin{aligned}
P_{s}(x) & =\frac{16 \mathcal{N}}{\sigma^{2}} \exp \left(-2 \ln \left(x^{4}-x^{2}+8\right)+\int^{x} \frac{32}{\sigma^{2}} \frac{\left(z-z^{3}\right)}{\left(z^{4}-z^{2}+8\right)^{2}}+\alpha \frac{4 z^{3}-8 z}{z^{4}-z^{2}+8} d z\right) \\
& =\frac{16 \mathcal{N}}{\sigma^{2}} \exp \left(2(\alpha-1) \ln \left(x^{4}-x^{2}+8\right)+\frac{1}{\sigma^{2}}\left(\tan ^{-1}\left(1-\frac{x^{2}}{2}\right)-\frac{2\left(x^{2}-6\right)}{\left(x^{4}-x^{2}+8\right)}\right)+C\right)
\end{aligned}
$$




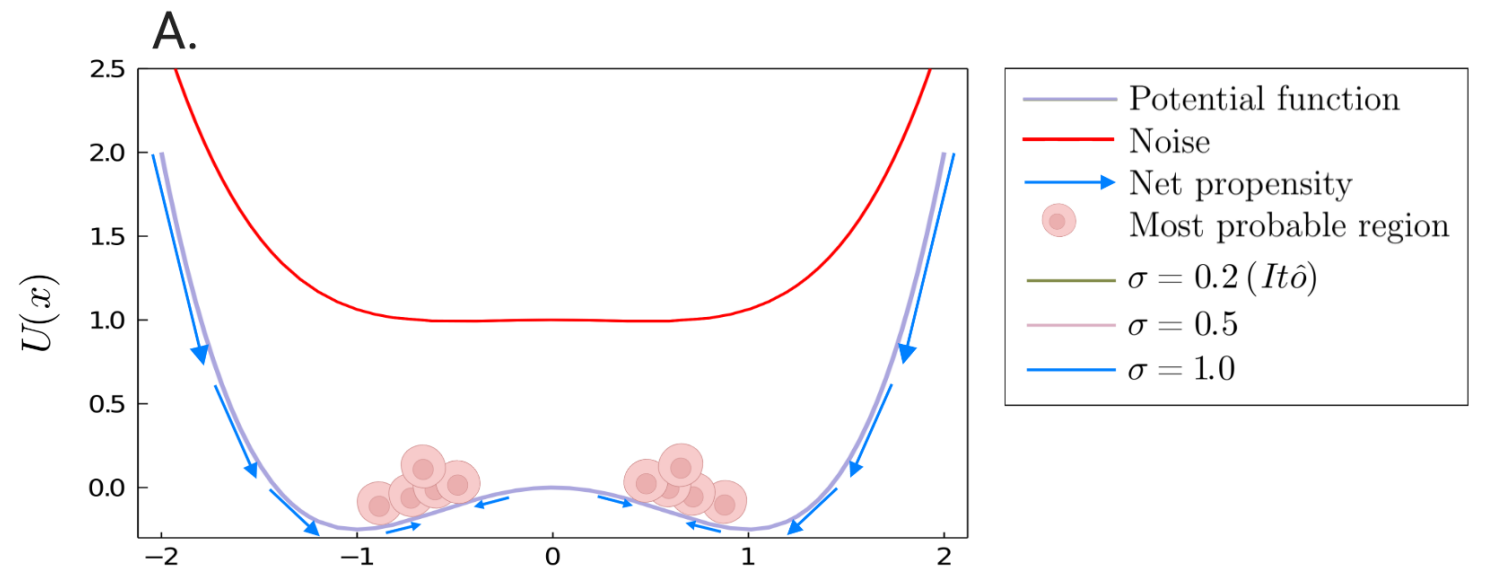

B.
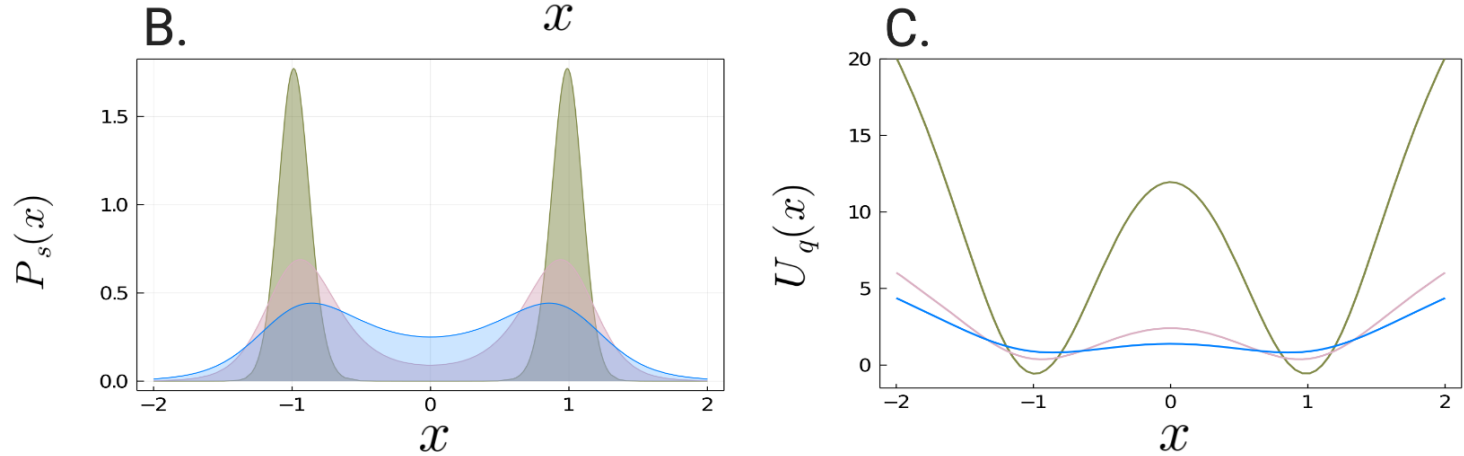

Figure 2: (A) A schematic of the system's trajectory. We consider the deterministic double-well potential defined in Eq. (8) of the main text driven by multiplicative noise. The schematic is plotted for $\sigma=0.5$ Cells are driven towards the fixed points of the noise curve $g(x)\left(x_{\min }= \pm \frac{1}{2}\right)$. (B\&C) Analytical steady-state probability distribution $P_{S}(x)$ given by Eq. 17) for the Itô interpretation only. We consider the effects of increasing strengths of multiplicative noise $g(x)=\frac{\sigma}{8}\left(2 x^{4}-x^{2}+16\right)$, where $\sigma=0.2$ (green curve), 0.5 (pink curve), 1.0 (blue curve). As $\sigma$ increases, the maxima of $P_{s}(x)$ and minima of $U_{q}(x)$ drift towards origin, approaching $x= \pm \frac{1}{2}$.

where $\mathcal{N}$ is the normalisation constant and $C$ is the constant of integration. It then follows immediately from Eq. (7) in the main text, that

$$
U_{q}(x)=\ln \left(\frac{\sigma^{2}}{16 \mathcal{N}}\left(x^{4}-x^{2}+8\right)^{2(1-\alpha)}\right)-\frac{1}{\sigma^{2}}\left(\tan ^{-1}\left(1-\frac{z^{2}}{2}\right)-\frac{2\left(z^{2}-6\right)}{\left(z^{4}-z^{2}+8\right)}\right)-C
$$

In Fig. (3) in the main text, the value for $C$ is $\frac{1}{\sigma^{2}}\left(\tan ^{-1}(1)+\frac{3}{2}\right)+2 \alpha \ln (8)$, and the normalisation constant is calculated accordingly.

In the main text, we discussed an additional example that behaves in a similar way to that of Example 1, but shifts the fixed points of the system towards the origin (as opposed to being pushed away from the origin as in Example 1). We provide here details of this example. If we consider the noise component $g(x)=\frac{\sigma}{8}\left(2 x^{4}-x^{2}+8\right)$ on the double-well potential $U(x)$ (defined in Eq. (8) of the main text), then it can be shown that the steady-state probability distribution of the state of the system $x$ is given by

$$
P_{s}(x)=\frac{64 \mathcal{N}}{\sigma^{2}} \exp \left(-2 \ln \left(2 x^{4}-x^{2}+8\right)+\frac{128}{\sigma^{2}}\left(\frac{1}{63 \sqrt{7}} \tan ^{-1}\left(\frac{4 x^{2}-1}{3 \sqrt{7}}\right)+\frac{x^{2}+5}{84 x^{4}-42 x^{2}+336}+C\right)\right),
$$

where $\mathcal{N}$ is the normalisation constant and $C$ is the constant of integration. In Fig. 2(A), we plot the effects of the noise $g(x)$ (for $\sigma=0.5$ ) on the double-well potential $U(x)$. We can see from Fig. 2(B\&C) that as the noise intensity $\sigma$ increases, the fixed points of steady-state distribution $P_{s}(x)$ and corresponding probabilistic landscape $U_{q}(x)$, shift towards those of the noise curve $g(x)$, which occur at $\left.x_{\min }= \pm \frac{1}{2}\right)$. 


\section{Example 2: Destruction of fixed points}

We provide full details of the derivations to the stationary probability distribution and probabilistic landscape given in Example 2 of the main text. We also derive the stationary points of the probabilistic landscape, which we use to calculate the critical noise at which the stochastic system transitions from bistable to monostable.

Derivation of the steady-state distribution and probabilistic landscape

From the general solution of the steady-state probability distribution (Eq. (6)), with $f(x)=x-x^{3}$ and $g(x)=\sigma\left(1+x^{2}\right)(\sigma>0)$, we have

$$
P_{s}(x)=\frac{\mathcal{N}}{\sigma^{2}\left(1+x^{2}\right)^{2}} \exp \left(2 \int^{x} \frac{z-z^{3}+2 \alpha \sigma^{2} z\left(1+z^{2}\right)}{\sigma^{2}\left(1+z^{2}\right)^{2}} d z\right)
$$

where $\mathcal{N}$ is the normalisation constant. We consider the integral in Eq. (18) which, after using partial fractions, can be written as

$$
\frac{2}{\sigma^{2}} \int^{x} \frac{2 z}{\left(1+z^{2}\right)^{2}}+\left(2 \alpha \sigma^{2}-1\right) \frac{z}{1+z^{2}} d z
$$

Now, letting $u=z^{2}$ so that $z=\frac{1}{2} \frac{d u}{d z}$, we obtain

$$
\frac{2}{\sigma^{2}} \int_{0}^{x^{2}} \frac{1}{(1+u)^{2}} d u+\frac{\left(2 \alpha \sigma^{2}-1\right)}{2} \frac{1}{(1+u)} d u .
$$

Then integrating with respect to $u$ we obtain,

$$
\frac{1}{\sigma^{2}}\left(\left(2 \alpha \sigma^{2}-1\right) \ln \left(\left|1+x^{2}\right|\right)-\frac{2}{\left(1+x^{2}\right)}+C\right),
$$

where $C$ is the constant of integration. It follows that

$$
P_{s}(x)=\frac{\mathcal{N}}{\sigma^{2}\left(1+x^{2}\right)^{2}} \exp \left(\frac{1}{\sigma^{2}}\left(\left(2 \alpha \sigma^{2}-1\right) \ln \left(1+x^{2}\right)-\frac{2}{\left(1+x^{2}\right)}+C\right)\right) .
$$

This is Eq. (13) in the main text. Now computing the probabilistic landscape, we immediately obtain

$$
U_{q}(x)=\ln \left(\frac{\sigma^{2}\left(1+x^{2}\right)^{2}}{\mathcal{N}}\right)-\frac{1}{\sigma^{2}}\left(\left(2 \alpha \sigma^{2}-1\right) \ln \left(1+x^{2}\right)-\frac{2}{\left(1+x^{2}\right)}+C\right),
$$

which is Eq. (14) in the main text. Note that for Fig. 4 in the main text we choose $C=2$, and the normalisation constant is calculated accordingly.

Stable stationary solutions of the probabilistic landscape

Differentiating $U_{q}(x)$ (as given by Eq. 20 above) with respect to $x$ and setting $U_{q}^{\prime}(x)=0$, we obtain (after simplification),

$$
x\left[x^{2}\left(4 \sigma^{2}-2\left(2 \alpha \sigma^{2}-1\right)+4 \sigma^{2}-4-2\left(2 \alpha \sigma^{2}-1\right)\right]=0 .\right.
$$

This implies that either $x=0$ or

$$
x^{2}=\frac{1-2 \sigma^{2}(1-\alpha)}{1+2 \sigma^{2}(1-\alpha)},
$$

which gives rise to Eq. (19) in the main text. 


\section{Identifiability considerations}

Decomposing the stationary probability distribution

The stationary probability distribution $P_{s}(x)$ of the SDE given in Eq. (1) is governed by the FPE given in Eq. (3), and can be seen to reduce to the differential equation given in Eq. (4), provided the detailed balance condition holds. We can use the differential equation given in Eq. Eq. (4) to uniquely solve for any one of the components $f(x), g(x)$ or $P_{s}(x)$ in terms of the other two; the uniqueness follows from the uniqueness property of first-order differential equations [2].

We begin by fixing a stochastic integration convention $\alpha$. Then given any stationary probability distribution $P_{s}(x)$ and noise component $g(x)$, the deterministic component $f(x)$ can be easily solved by rearranging the differential equation Eq. (4). We obtain

$$
f(x)=\frac{1}{P_{s}(x)}\left[\frac{\partial}{\partial x}\left(\frac{g^{2}(x) P_{s}(x)}{2}\right)\right]-\alpha g(x) g^{\prime}(x) .
$$

This is Eq. (18) in the main text. Now, given any stationary probability distribution $P_{s}(x)$ and deterministic component $f(x)$, the stochastic component $g(x)$ can be solved from the differential equation Eq. (4) as,

$$
g(x)= \begin{cases}{\left[\frac{2}{1-\alpha}\left(P_{s}(x)\right)^{\frac{1}{(\alpha-1)}} \int^{x} f(z)\left(P_{s}(z)\right)^{\frac{-1}{(\alpha-1)}} d z\right]^{\frac{1}{2}}} & \text { for } \alpha \in[0,1) \\ {\left[\frac{2}{P_{s}^{\prime}(x)} P_{s}(x) f^{\prime}(x)\right]^{\frac{1}{2}}} & \text { for } \alpha=1 .\end{cases}
$$

which is Eq. (19) in the main text. We now check that this is the solution to differential equation given in Eq. (4). It easy to see that Eq. (4) is equivalent to

$$
\frac{1}{2} g^{2}(x) P_{s}^{\prime}(x)=f(x) P_{s}(x)+(\alpha-1) g(x) g^{\prime}(x) P_{s}(x),
$$

We consider two cases. First consider the case where $\alpha=1$. It follows immediately from Eq. (23), that

$$
g(x)=\left[\frac{2}{P_{s}^{\prime}(x)} P_{s}(x) f^{\prime}(x)\right]^{\frac{1}{2}} .
$$

Now assume that $\alpha \in[0,1)$. Then from Eq. (23), we have

$$
\begin{array}{r}
g^{\prime}(x)=\frac{1}{2}\left[\frac{2}{1-\alpha}\left(P_{s}(x)\right)^{\frac{1}{(\alpha-1)}} \int^{x} f(z)\left(P_{s}(z)\right)^{\frac{-1}{(\alpha-1)}} d z\right]^{\frac{-1}{2}} \\
{\left[\frac{2}{(1-\alpha)(\alpha-1)}\left(P_{s}(x)\right)^{\frac{1}{(\alpha-1)}-1} P_{s}^{\prime}(x) \int^{x} f(z)\left(P_{s}(z)\right)^{\frac{-1}{\alpha-1}} d z\right] .}
\end{array}
$$

So that,

$$
(\alpha-1) g(x) g^{\prime}(x)=\frac{1}{1-\alpha}\left(P_{s}(x)\right)^{\frac{1}{(\alpha-1)}} P_{s}^{\prime}(x) \int^{x} f(z)\left(P_{s}(z)\right)^{\frac{-1}{\alpha-1}} d z-f(x) P_{s}(x) .
$$

Thus, the right hand side of Eq. (24) is equal to

$$
\frac{1}{1-\alpha}\left(P_{s}(x)\right)^{\frac{1}{(\alpha-1)}} P_{s}^{\prime}(x) \int^{x} f(z)\left(P_{s}(z)\right)^{\frac{-1}{\alpha-1}} d z,
$$

which equals the left hand side of Eq. (24). Hence $g(x)$ is indeed the solution to differential equation given in Eq. 4. 


\section{Non-identifiability of the first kind}

Here we derive the stationary probability distribution $P_{s}(x)$ (Eq. (20) of the main text) and deterministic component $f_{2}(x)=2 x-\frac{1}{4} x \exp \left(2 x^{2}\right)$ used in the main text to demonstrate non-identifiabilty of the first kind. We begin by deriving $P_{s}(x)$. From Eq. (6), with $f_{1}(x)=-x$ and $g_{1}(x)=\sigma \exp \left(-x^{2}\right)$, we have

$$
\begin{aligned}
P_{s}(x) & =\frac{\mathcal{N}}{\sigma^{2}} \exp \left(2 x^{2}-\frac{2}{\sigma^{2}} \int^{x} z \exp \left(2 z^{2}\right) d z\right) \\
& =\frac{\mathcal{N}}{\sigma^{2}} \exp \left(2 x^{2}-\frac{1}{2 \sigma^{2}} \exp \left(2 x^{2}\right)+C\right),
\end{aligned}
$$

where $\mathcal{N}$ is the normalisation constant and $C$ is the constant of integration. Now, using Eq. 22), with $g_{2}(x)=1$ and $P_{s}(x)$, the deterministic component can be solved as

$$
f_{1}(x)=\frac{1}{P_{s}(x)} \frac{\partial}{\partial x}\left(\frac{P_{s}(x)}{2}\right)=2 x-\frac{1}{\sigma^{2}} \exp \left(2 x^{2}\right) x
$$

\section{References}

[1] C. W. Gardiner, Handbook of stochastic methods, Springer Berlin, 1985.

[2] V. I. Arnold, Ordinary differential equations, The MIT Press, 1998. 